\section{YAP controls retinal stem cell DNA replication timing and genomic stability}

\begin{abstract}
Pauline Cabochette ${ }^{1 \dagger}$, Guillermo Vega-Lopez ${ }^{1 \dagger}$, Juliette Bitard ${ }^{1,2}$, Karine Parain ${ }^{3}$, Romain Chemouny ${ }^{1}$, Christel Masson ${ }^{1,2}$, Caroline Borday ${ }^{1}$, Marie Hedderich ${ }^{3}$, Kristine A Henningfeld ${ }^{3}$, Morgane Locker ${ }^{1}$, Odile Bronchain ${ }^{1 *}$, Muriel Perron ${ }^{1,2,4 *}$
\end{abstract}

${ }^{1}$ Paris-Saclay Institute of Neuroscience, CNRS, Université Paris Sud, Orsay, France; ${ }^{2}$ Centre d'Etude et de Recherche Thérapeutique en Ophtalmologie, Retina France, Orsay, France; ${ }^{3}$ Center for Nanoscale Microscopy and Molecular Physiology of the Brain, Institute of Developmental Biochemistry, University of Goettingen, Goettingen, Germany; ${ }^{4}$ Jules Stein Eye Institute, University of California Los Angeles, Los Angeles, United States
*For correspondence: odile. bronchain@u-psud.fr (OB); muriel.perron@u-psud.fr (MP)

tThese authors contributed equally to this work

Competing interests: The authors declare that no competing interests exist.

Funding: See page 20

Received: 02 May 2015

Accepted: 20 August 2015

Published: 22 September 2015

Reviewing editor: Helen McNeill, The Samuel Lunenfeld Research Institute, Canada

(c) Copyright Cabochette et al. This article is distributed under the terms of the Creative Commons Attribution License, which permits unrestricted use and redistribution provided that the original author and source are credited.

\begin{abstract}
The adult frog retina retains a reservoir of active neural stem cells that contribute to continuous eye growth throughout life. We found that Yap, a downstream effector of the Hippo pathway, is specifically expressed in these stem cells. Yap knock-down leads to an accelerated $\mathrm{S}$-phase and an abnormal progression of DNA replication, a phenotype likely mediated by upregulation of $c-M y c$. This is associated with an increased occurrence of DNA damage and eventually p53-p21 pathway-mediated cell death. Finally, we identified PKNOX1, a transcription factor involved in the maintenance of genomic stability, as a functional and physical interactant of YAP. Altogether, we propose that YAP is required in adult retinal stem cells to regulate the temporal firing of replication origins and quality control of replicated DNA. Our data reinforce the view that specific mechanisms dedicated to S-phase control are at work in stem cells to protect them from genomic instability.
\end{abstract}

DOI: 10.7554/eLife.08488.001

\section{Introduction}

Adult stem cell maintenance is required to sustain long-term preservation of tissue homeostasis. In the fish or amphibian retina, a continuously proliferating peripheral domain called ciliary marginal zone (CMZ) (Wetts et al., 1989; Perron et al., 1998) was recently formally demonstrated to contain genuine multipotent and self-renewing neural stem cells (Centanin et al., 2011). The CMZ not only ensures cell replacement, but also contributes to life-long growth of the eye through the permanent generation of all retinal cell types. The CMZ thus represents an ideal model for dissecting molecular cues underlying retinal stem cell properties in vivo. Such knowledge is essential for the development of innovative therapeutic strategies based on the mobilization and targeted activation of endogenous neural stem cells for tissue repair.

The Hippo pathway effector yes-associated protein (YAP) was identified as a major regulator of organ growth through its actions on embryonic precursor cells (Lian et al., 2010; Ramos and Camargo, 2012). YAP function in adult stem cells, however, remains unclear. For instance, Yap overexpression increases self-renewal of airway basal stem cells (Zhao et al., 2014). In contrast, it surprisingly leads to a loss of intestinal stem cells (Barry et al., 2013), while being seemingly neutral regarding the quantity and function of hematopoietic stem cells (Jansson and Larsson, 2012). Inactivation studies further suggested that YAP is largely dispensable in a physiological context for the homeostasis of several adult organs (Cai et al., 2010; Azzolin et al., 2014; Chen et al., 2014; Zhang et al., 2014), although this might reflect in some cases functional redundancy with the other Hippo effector TAZ 
eLife digest In animals, stem cells divide to produce the new cells needed to grow and renew tissues and organs. Understanding the biology of these cells is of the utmost importance for developing new treatments for a wide range of human diseases, including neurodegenerative diseases and cancer. Before a stem cell divides, it copies its DNA and the two sets of genetic instructions are then separated so that the two daughter cells both have a complete set. This process needs to be as accurate as possible because any errors would result in incorrect genetic information being passed on to the daughter cells.

Stem cells in the light-sensitive part of the eye-called the retina-divide to produce the cells that detect light and relay visual information to the brain. In many animals, these stem cells stop dividing soon after birth and the retina stops growing. However, the stem cells in frogs and fish continue to divide throughout the life of the animal, which enables the eye to keep on growing.

A protein called YAP regulates the growth of organs in animal embryos, but it is not clear what role this protein plays in stem cells, particularly after birth. To address this question, Cabochette et al. studied YAP in the retina of frog tadpoles. The experiments show that YAP is produced in the stem cells of the retina after birth and is required for the retina to continue to grow.

Cabochette et al. used tools called 'photo-cleavable Morpholinos' to alter the production of YAP in adult stem cells. The cells that produced less YAP copied their DNA more quickly and more of their DNA became damaged, which eventually led to the death of these cells. Further experiments revealed that YAP interacts with a protein called PKNOX1, which is involved in maintaining the integrity of DNA.

Cabochette et al.'s findings provide the first insights into how YAP works in the stem cells of the retina and demonstrate that it plays a crucial role in regulating when DNA is copied. A future challenge is to find out whether YAP plays a similar role in the stem cells of other organs in adult animals.

DOI: 10.7554/eLife.08488.002

(Imajo et al., 2015). YAP is implicated in tissue regeneration but its effects are controversial (Cai et al., 2010; Barry et alo, 2013). Thus, the role of YAP in vertebrate adult stem cells may likely be context-dependent and clearly deserves further investigation. Since its function in adult neural stem cells is presently unknown, we took advantage of the Xenopus CMZ model system and investigated whether Yap is involved in the maintenance of an active pool of retinal stem cells in the continuously growing post-embryonic frog eye. Although YAP gain of function led quite expectedly to CMZ cell overproliferation, the loss of function analysis revealed a more complex phenotype. Indeed, we found that stem cells were still present but exhibited aberrant cell cycle progression. In particular, DNA replication timing was found to be altered leading to a dramatic S-phase shortening. This correlates with increased DNA damage and eventually cell death. We also found that YAP functionally and physically interacts with PKNOX1, a transcription factor required to maintain genomic stability (lotti et al., 2011).

\section{Results}

\section{Yap is expressed in slow dividing stem cells of the post-embryonic retina}

In situ hybridization at the optic vesicle stage revealed prominent Yap expression in the presumptive retinal pigmented epithelium (RPE) and in the neural retina/RPE border (Figure 1-figure supplement 1A), a region we previously proposed to be the presumptive adult stem cell niche (El Yakoubi et al., 2012). In line with this, we found that in the post-embryonic retina, Yap is expressed in the most peripheral stem cell-containing region of the CMZ (Figure 1A,B). We also performed immunostaining using an antibody whose specificity was assessed in a loss of function context, that is, in tadpoles injected with Yap Morpholinos (Yap-MO; Figure 1-figure supplement 2; see also Figure 2-figure supplement 1 for efficiency and specificity evaluation of Yap-MO). YAP protein was detected in stem cells located at the tip of the CMZ, mainly in the cytoplasm, although some signal could be observed as well in the nuclei of these cells. Of note, we also found YAP labeling in Müller glial cells (Figure 1C). To delineate more precisely the Yap expression domain, we co-labeled Yap and proliferative cells (Figure 1D). A short EdU pulse was performed allowing slow dividing stem cells to be distinguished from fast 

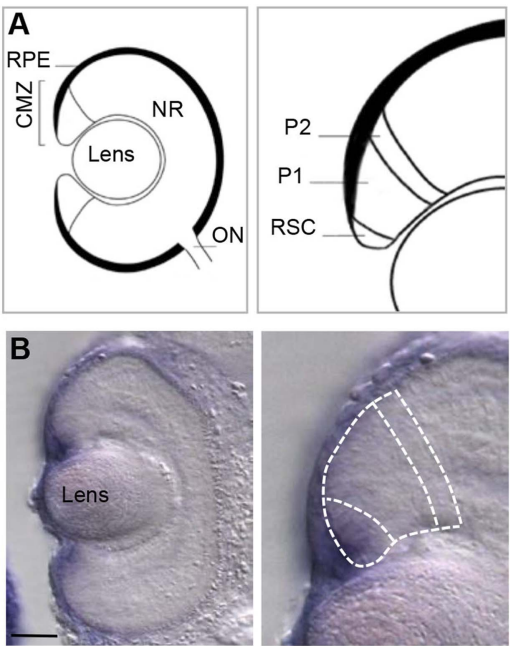

E
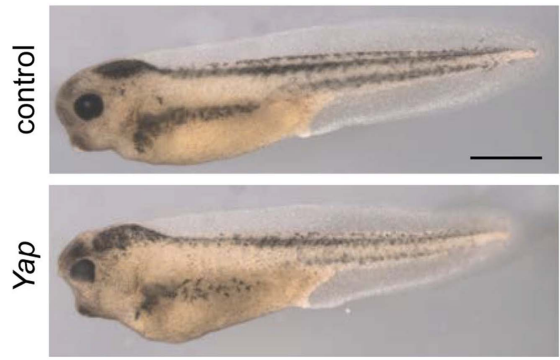

G

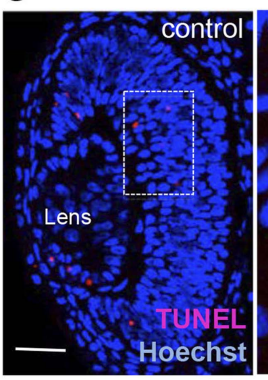

I
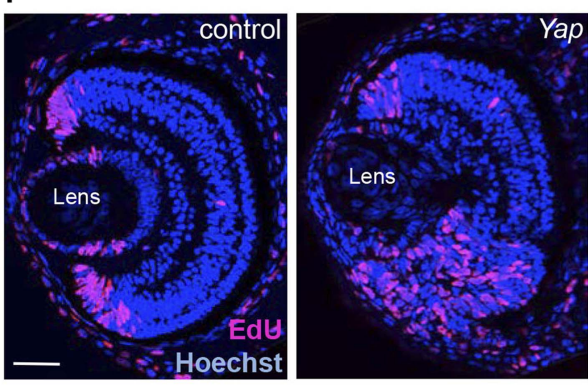
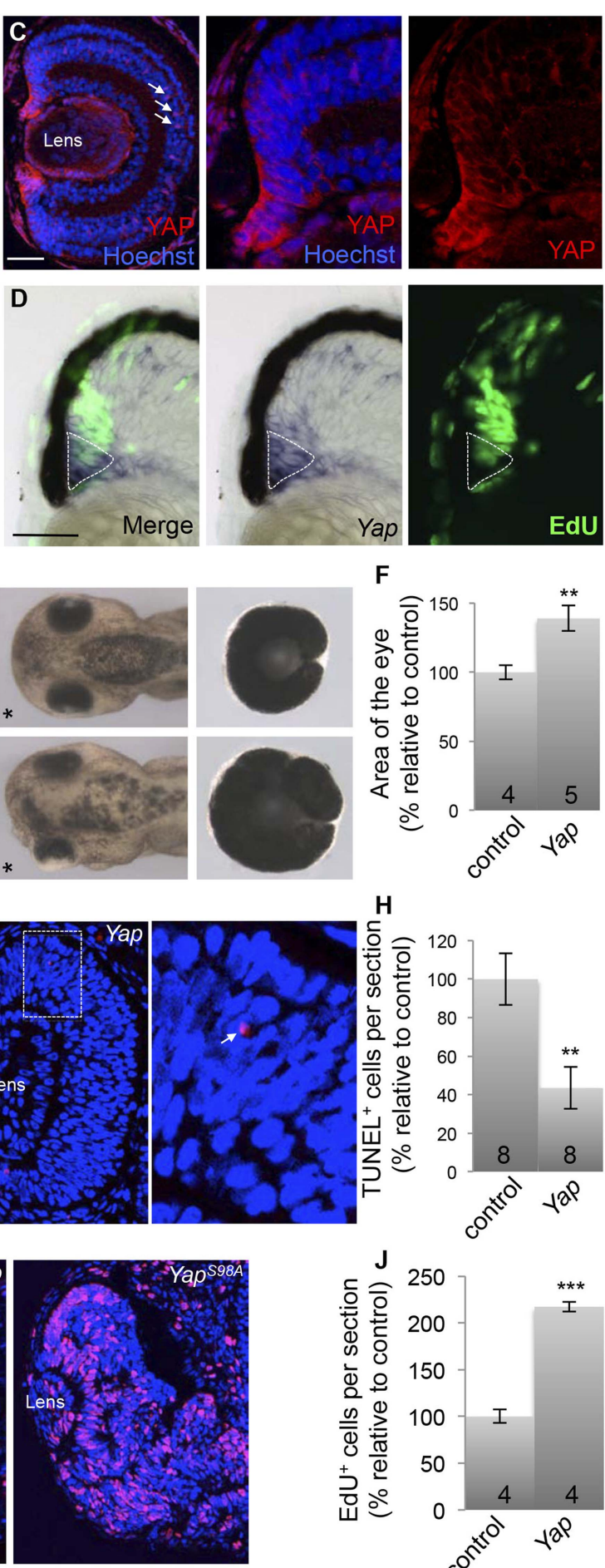

$\mathbf{F}$
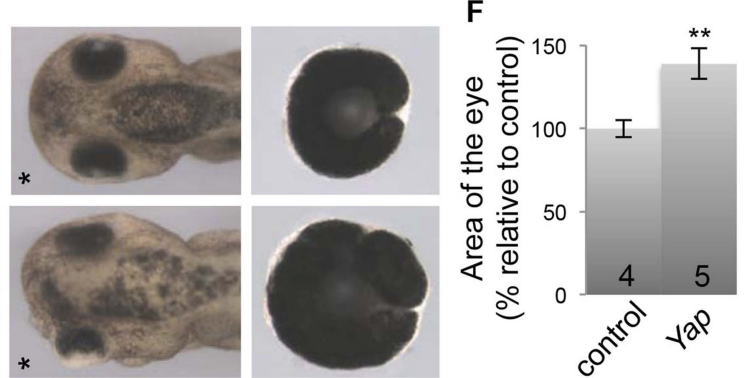

$\mathbf{H}$
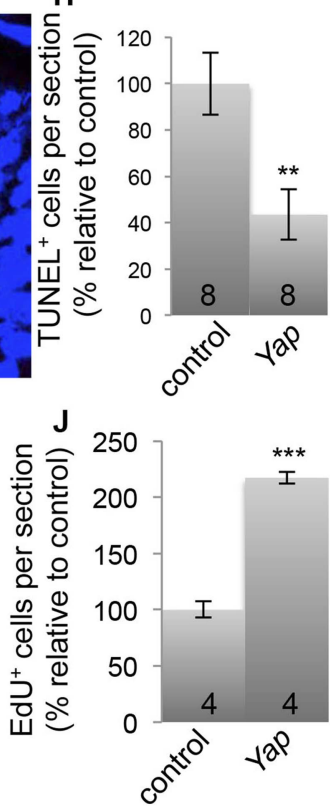

Figure 1. Yap overexpression expands the proliferating cell population in the post-embryonic retina. (A) Schematic transversal section of a Xenopus tadpole retina (RPE: retinal pigment epithelium; NR: neural retina; ON: optic nerve). Within the CMZ (right panel), retinal stem cells (RSC) reside in the most peripheral margin while actively dividing progenitors (P1) and their post-mitotic progeny (P2) are localized more centrally. (B) In situ hybridization analysis of Yap expression on stage 40 retinal sections. The image on the right is a higher magnification of the CMZ (dashed lines represent the different zones as in a). (C) Immunostaining with anti-YAP antibody on stage 42 retinal sections. YAP labeling is detected in the CMZ as well as in Müller glial cells (arrows). Images on the right are higher

Figure 1. continued on next page 
Figure 1. Continued

magnifications of the CMZ. (D) EdU labeling (3-hr pulse) following in situ hybridization with a Yap probe (dotted line) on stage 40 retinal sections. (E) Lateral views (left panels), head dorsal views (middle panels) and dissected eyes (right panels) of stage 40 tadpoles following two-cell stage microinjection of GFP mRNA as a lineage tracer with either B-gal (control) or Yap mRNA. The asterisk indicates the injected side. (F) Quantification of dissected eye area. (G-J) TUNEL (G, H; stage 33/34) or EdU incorporation (I, J; 3-hr pulse at stage 40) assays analyzed on retinal sections from tadpoles injected as in (E). Arrows point to TUNEL-positive cells in higher magnifications of the area delineated with dotted line $(\mathbf{G})$. The number of analyzed retinas is indicated in each bar. Data are represented as mean \pm SEM. Scale bar $=1 \mathrm{~mm}$ in $(\mathbf{E})$ and $40 \mu \mathrm{m}$ in other panels.

DOI: 10.7554/eLife.08488.003

The following figure supplements are available for figure 1:

Figure supplement 1. Yap, Taz and Tead expression.

DOI: 10.7554/eLife.08488.004

Figure supplement 2. Validation of YAP antibody specificity.

DOI: 10.7554/eLife.08488.005

Figure supplement 3. Yap ${ }^{\Delta T B S}$ does not promote $C M Z$ cell proliferation.

DOI: 10.7554/eLife.08488.006

proliferating transit amplifying progenitors in the CMZ (Xue and Harris, 2011). Yap staining was found to be prominent in EdU-negative stem cells and in the most peripheral EdU-positive cells (young progenitors). The staining then waned in more central older progenitor cells. Of note, in contrast to Yap, Taz is faintly expressed in the post-embryonic retina and only a weak and diffuse signal could be detected in the CMZ (Figure 1-figure supplement 1B).

Finally, as YAP acts as a co-transcriptional activator, we wondered whether its classical partners of the TEAD family were also expressed in the CMZ. We found consistent labeling of both Tead1 and Tead2 in the periphery of the CMZ where Yap is expressed (Figure 1-figure supplement 1C).

\section{Yap overexpression promotes post-embryonic eye overgrowth}

To investigate YAP function in the post-embryonic retina, we first undertook a gain of function approach by the means of mRNA injection at the two-cell stage. Yap-overexpressing tadpoles displayed eye overgrowth on the injected side (Figure 1E,F). This phenotype prompted us to analyze the impact of YAP on both cell death and proliferation. We found that Yap overexpression results in both a decreased number of TUNEL-positive cells (Figure $1 G, H$ ) and a dramatic expansion of the EdU-positive cell population (Figure 11,J). The overproliferative phenotype was strongly exacerbated upon overexpression of a Yap mutant construct where Ser-98 was replaced by an alanine (Yap ${ }^{\mathrm{S98A}}$ ) (Figure 1I). This residue (Ser-127 in mammalian YAP) is a conserved Lats phosphorylation site that has been shown to mediate the growth-suppressive output of the Hippo signaling cascade in vivo (Zhao et al., 2007). In contrast, overexpression of a truncated construct lacking the TEAD binding site (Yap ${ }^{\triangle T B S}$ ) was unable to trigger enhanced proliferation in the $\mathrm{CMZ}$, suggesting that the overproliferative phenotype requires interaction with a TEAD protein (Figure 1-figure supplement 3). Together, these data reveal that Yap-dependent retinal overgrowth is likely caused by enhanced cell survival and cell proliferation.

\section{Yap knockdown reduces post-embryonic eye size}

We next sought to determine whether Yap is essential for post-embryonic retinal growth by knocking it down using Yap-MO. The Morpholino concentration was chosen to efficiently decrease YAP quantity (as inferred from Western-blot analysis; Figure 2-figure supplement 1A), while avoiding previously described early embryonic defects (Gee et al., 2011). In these conditions, morphant tadpoles developed but exhibited significantly reduced eye size compared to controls (Figure 2A,B). Importantly, this phenotype was restored upon co-injection of Yap-MO with non-targetable Yap mRNAs, demonstrating specificity (Figure 2-figure supplement 1B,C). To exclude potential growth impairment at the level of the whole organism and assess the tissue autonomy of eye size defects, we performed optic vesicle isotopic and isochronic graft experiments (Figure 2C). When the optic vesicle of a control tailbud was transplanted into an enucleated morphant embryo, it nevertheless reached a normal size. In contrast, Yap-MO optic vesicles grafted in a control host generated smaller eyes, which is in accordance with Yap knockdown effects being eye autonomous. 

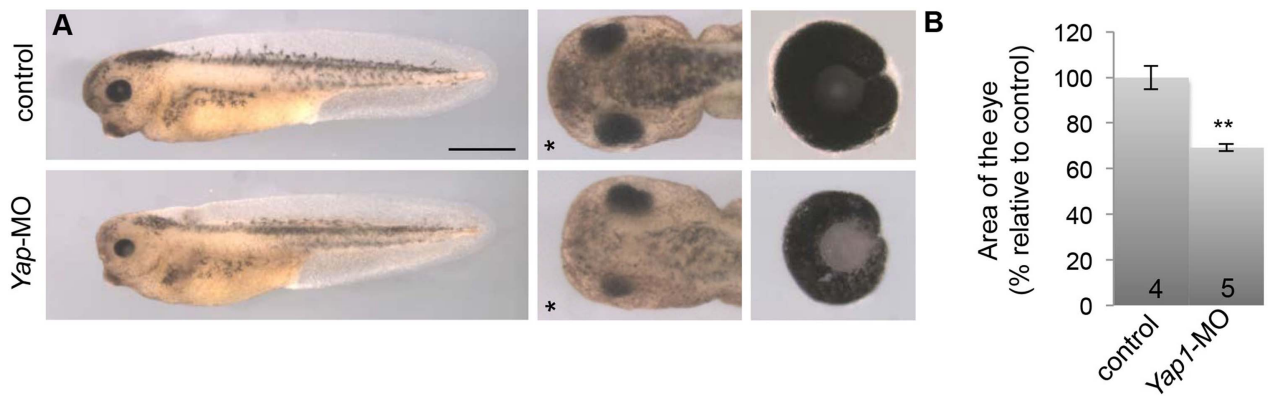

C
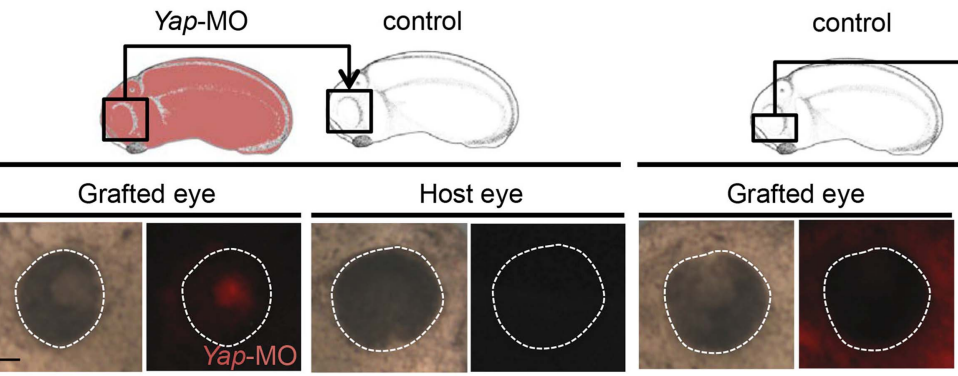

Yap-MO

D

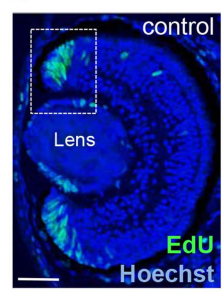

$\mathrm{F}$

F $\quad H e s 4$

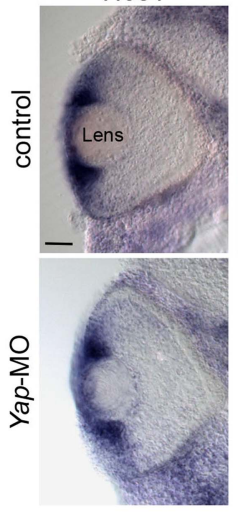

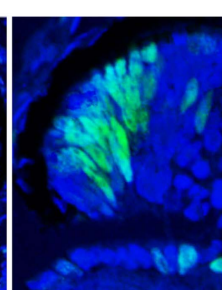

Atoh7
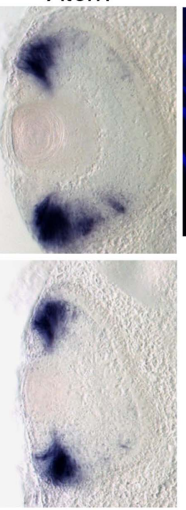

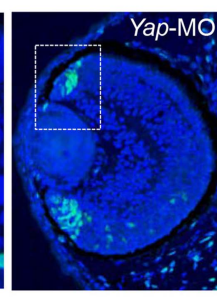

G

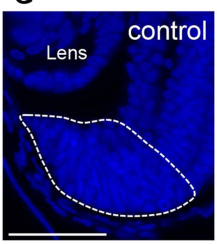

H

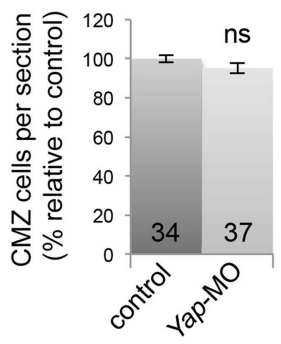

E 120 control Yap-MO
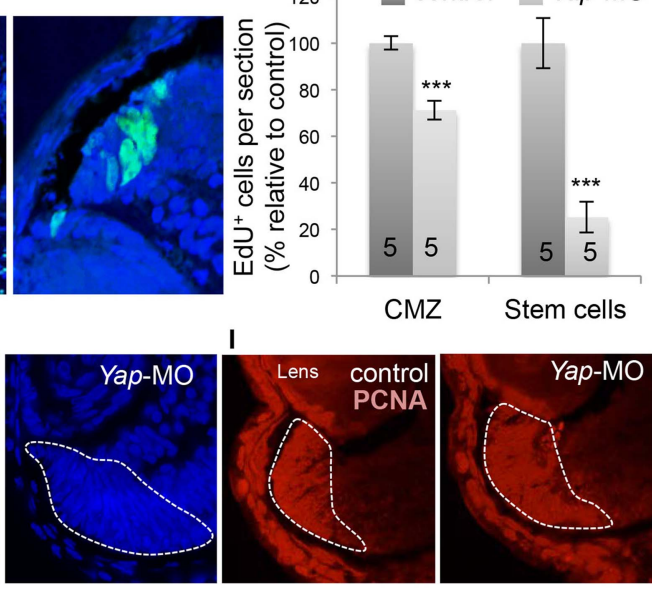

J

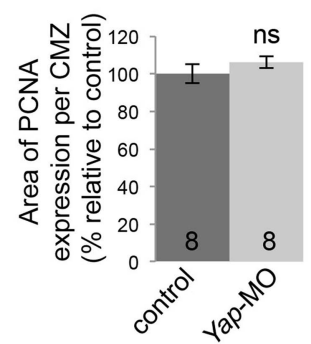

Figure 2. Yap knockdown decreases eye size and EdU incorporation in the post-embryonic retina. (A) Lateral views (left panels), head dorsal views (middle panels) and dissected eyes (right panels) of stage 40 tadpoles following two-cell stage microinjection of Yap-5-mismatch-MO (control) or Yap-MO. The asterisk indicates the injected side. (B) Quantification of dissected eye area. (C) Eyes of stage 40 tadpoles following optic vesicle transplantation at stage 25 as shown in the schematic. Dotted lines delineate the eye circumference. (D) EdU incorporation assay (6-hr pulse) analyzed on retinal sections from stage 40 tadpoles injected as in (A). Higher magnifications of the CMZ (delineated by dotted lines) are shown for each condition. (E) Quantification of EdU-positive cells within the whole CMZ or within the most peripheral stem cell compartment. (F) In situ hybridization analysis of Hes4 (retinal stem cell marker; El Yakoubi et al., 2012) and Atoh7 (progenitor cell marker; Kanekar et al., 1997) expression on stage 40 retinal Figure 2. continued on next page 
Figure 2. Continued

sections from tadpoles injected as in (A). (G-J) Hoechst staining and PCNA immunolabeling on stage 40 retinal sections from tadpoles injected as in (A). The CMZ is delineated with dotted lines. The number of analyzed retinas per condition is indicated in each bar. Data are represented as mean \pm SEM. Scale bar $=1 \mathrm{~mm}$ in (A) and $40 \mu \mathrm{m}$ in other panels.

DOI: 10.7554/eLife.08488.007

The following figure supplement is available for figure 2 :

Figure supplement 1. Validation of Yap-MO efficiency and specificity. DOI: 10.7554/eLife.08488.008

Finally, to address whether the reduced eye size was due to abnormal embryonic morphogenesis or to post-embryonic growth defects, we adapted in Xenopus the use of photo-cleavable Morpholinos (photo-MO). This technology, previously set up in zebrafish (Tallafuss et al., 2012), allows for an inducible or reversible gene knockdown through UV-induced cleavage of either sense or antisense photo-MOs (Figure 3A). We found that restoring YAP function at late embryogenesis, following knockdown during development, leads to tadpoles with normal sized eyes (Figure 3B-D). This supports the hypothesis that the Yap-MO phenotype is not the indirect consequence of developmental morphogenetic defects. In line with this, conditional Yap knockdown starting at late retinogenesis was found to be sufficient to trigger a small eye phenotype (Figure $3 B, E, F$ ). Together, these data point to a specific role for YAP in the homeostatic control of post-embryonic retinal growth.

\section{Yap knockdown impedes retinal stem cell proliferative activity}

To investigate the cause of eye size reduction in Yap morphant tadpoles, we determined the level of proliferation within the CMZ. Yap-MO-injected tadpoles harbored a significantly decreased number of $\mathrm{EdU}^{+}$cells compared to a control situation (Figure 2D,E). The same was true when Yap knockdown was conditionally induced from late embryogenesis (Figure 3-figure supplement 1). The difference in EdU-labeling between control and Yap morphant tadpoles was even more pronounced at the tip of the $\mathrm{CMZ}$ where stem cells reside and Yap expression is the strongest (Figure 2E). We thus reasoned that Yap knockdown might decrease the number of proliferative cells in the $\mathrm{CMZ}$ as a consequence of stem cell depletion. Using in situ hybridization, we examined the expression of several retinal stem and progenitor cell markers (Figure 2F and data not shown). Surprisingly, stainings observed in Yap-MO-injected tadpoles were similar to control ones, indicating that both stem and progenitor cell populations were still present. Accordingly, neither the total number of cells within the CMZ nor the size of the proliferative cell population (PCNA labeled) was significantly affected (Figure 2G-J). Together, these data indicate that Yap knockdown does not induce stem/progenitor cell exhaustion but rather alters the relative proportion of time these cells spend in S-phase of the cell cycle.

\section{Yap loss of function affects cell cycle progression within the $\mathrm{CMZ}$}

The observed phenotype suggests that cell cycle kinetics of retinal stem/progenitor cells is perturbed in morphant tadpoles. As a first global approach to test this hypothesis, we evaluated the mitotic index in the whole post-embryonic CMZ using the mitotic marker phospho-histone H3 (PH3; Figure 4A,B). We found that compared to the control, Yap knockdown results in a significantly lower percentage of mitotic cells per section, which is suggestive of a longer total cell cycle length. To further investigate cell cycle progression defects at the level of the whole $\mathrm{CMZ}$, we then measured $\mathrm{G} 2$ length using the percentage of labeled mitosis (PLM) paradigm (Quastler and Sherman, 1959; Cai et al., 1997; Locker et al., 2006) (Figure 4C-E). As expected from the asynchrony among retinal cells in the CMZ, the percentage of $\mathrm{PH} / \mathrm{EdU}$-labeled cells increased sigmoidally with increasing EdU exposure times, before reaching a plateau (Figure 4E). Noticeably, the PLM was consistently lower at each time point in Yap morphant retinas compared to control ones, indicating a delayed S- to M-phase progression. We estimated that the mean $\mathrm{G} 2$ length ( $\mathrm{T}_{\mathrm{G} 2}$; see 'Materials and methods' for details) doubled in Yap morphant retinas compared to the control (from approximately 2.5 to $4.5 \mathrm{hr}$ ). It thus appears that Yap knockdown results in perturbed cell cycle kinetics within the CMZ. 
A

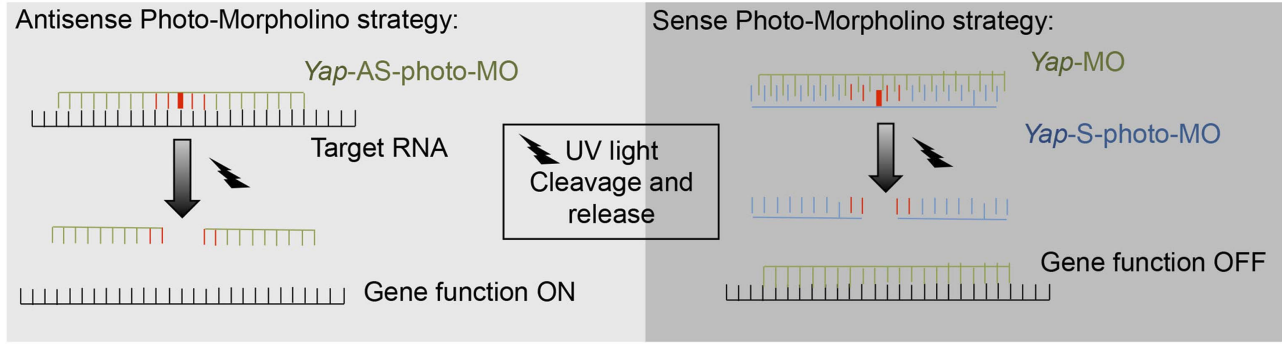

B

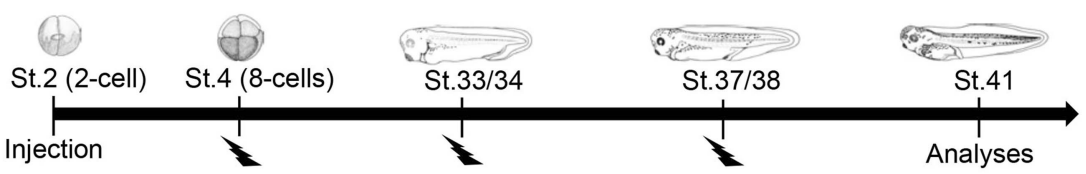

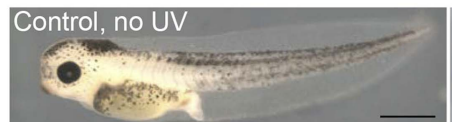

Yap-MO, no UV

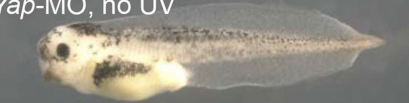

Yap-AS-photo-MO, no UV

$\theta$
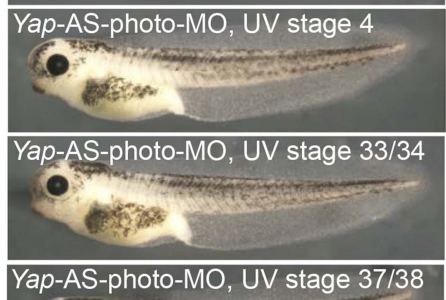

freture

6rostits

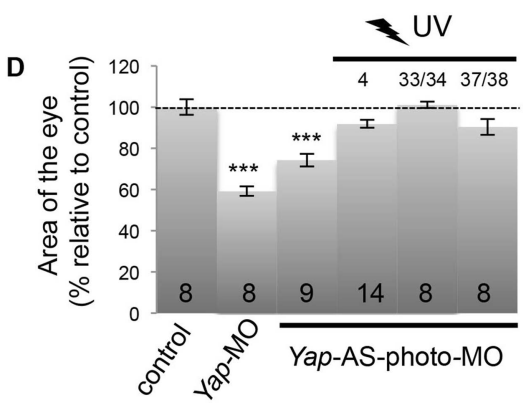

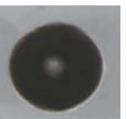
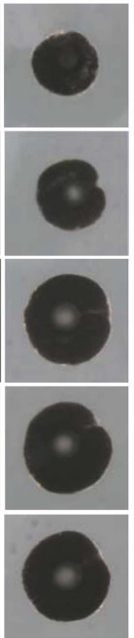

E
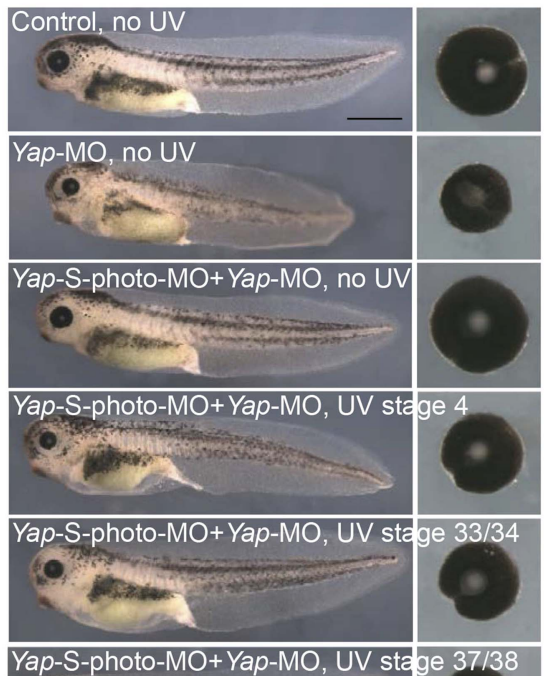

Yap-
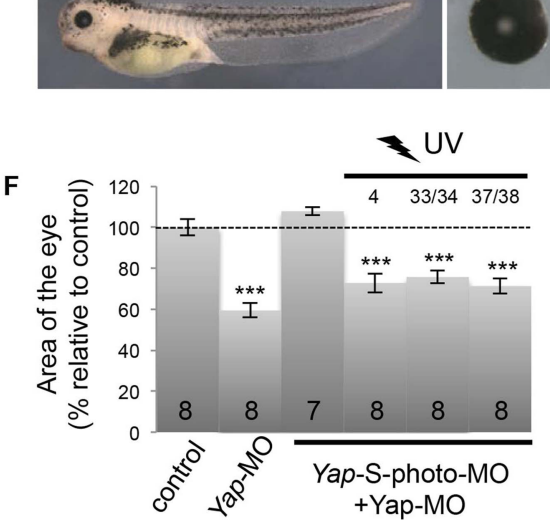

Figure 3. Conditional Yap knockdown in the retina. (A) Principle of reversible and inducible gene knockdown using photo-Morpholinos (photo-MO). Photo-MO contains a photo-sensitive subunit cleaved by $365 \mathrm{~nm}$ light. Yap-AS-photo-MO is degraded upon UV light exposure and its translation blocking activity is thus interrupted. Unmodified Yap-MO is rendered inactive by binding to Yap-S-photo-MO. It therefore cannot bind its mRNA target until light-induced cleavage of the sense MO. (B) Diagram of the experimental design. Embryos are microinjected with $\mathrm{MO}$ at the two-cell stage, subjected to UV exposure at different developmental stages as indicated (black flashes) and then sacrificed for analyses. (C-F) Analysis of reversible (C, D) and inducible (E, F) Yap knockdown. (C, E) Lateral views and dissected eyes of stage 41 tadpoles following two-cell stage microinjection of the indicated $\mathrm{MO}$ (see table in B). (D, F) Quantification of dissected eye area. The stage at which UV exposure was performed is indicated above each bar. The Yap-AS-photo-MO (without any UV exposure) shows the same efficiency Figure 3. continued on next page 
Figure 3. Continued

as the Yap-MO in reducing eye size. It is efficiently cleaved by UV light since exposure right after injection (stage 4) leads to a wild type phenotype. Restoring Yap function from stage 33/34 or even from stage 37/38 onwards leads to normal eye sized embryos, demonstrating that restricting Yap knockdown to embryogenesis is not sufficient to affect tadpole eye growth. The Yap-S-photo-MO efficiently blocks Yap-MO since their co-injection does not affect eye size. It is efficiently cleaved by UV light since exposure right after co-injection (stage 4) leads to a small eye phenotype, as observed in Yap-MO-injected embryos. Conditional Yap knockdown by light exposure from stage $33 / 34$ or even from stage 37/38 onwards is sufficient to reduce eye size, suggesting that Yap is required at postembryonic stages to maintain CMZ-dependent eye growth. Of note, in our experimental conditions, UV light exposure does not generate any significant effects on eye size (data not shown). The number of analyzed tadpoles is indicated within each bar. Scale bar $=1 \mathrm{~mm}$.

DOI: 10.7554/eLife.08488.009

The following figure supplement is available for figure 3 :

Figure supplement 1. Inducible Yap knockdown at post-embryonic stages reduces EdU incorporation in the CMZ. DOI: 10.7554/eLife.08488.010

\section{Yap loss of function affects S-phase duration in retinal stem cells}

In order to specifically measure total cycle length $\left(T_{C}\right)$ of retinal stem cells, we next turned to a cumulative labeling assay (Nowakowski et al., 1989), a well-established technique that also allows evaluating S-phase $\left(T_{S}\right)$ length (see 'Materials and methods' for details). As shown in Figure 4F, the labeling index in the linear part of the curve was consistently lower in Yap morphant retinal stem cells compared to control ones. Calculation of $\mathrm{T}_{\mathrm{C}}$ confirmed the hypothesis of extended cell cycle duration following Yap-Mo injection. Surprisingly, it also revealed a dramatic reduction of S-phase length in morphant cells (Figure 4G).

Such unexpected S-phase shortening prompted us to further investigate the underlying molecular mechanisms. In eukaryotes, origins of replication are activated throughout the S-phase in a temporally controlled manner such that some origins fire early and others fire late. The c-Myc proto-oncogene has been shown to accelerate S-phase by inducing premature origin firing initiation and increasing origin density (Robinson et al., 2009; Srinivasan et al., 2013). In situ hybridization and qPCR analyses revealed an upregulation and expansion of c-Myc expression in the $\mathrm{CMZ}$ of the Yap morphant retina (Figure 5A-C). This may account, at least in part, for the S-phase length shortening. To strengthen this hypothesis, we examined EdU-labeled replication foci at the tip of the CMZ. Their abundance and distribution, as classically observed in synchronized cultured cells, are indeed known to differ between early (numerous small foci located throughout the nucleus) and mid/late S-phase (limited number of large foci; Figure 5D) (van Dierendonck et al., 1989; Koberna et al., 2005). In the control situation, we found both early and late patterns within the stem cell population of the CMZ. In contrast, the proportion of cells in mid/late S-phase was dramatically reduced in Yap morphant cells (Figure 5E,F). Altogether, these data highlight that loss of YAP function in retinal stem cells alters their temporal program of DNA replication and points to $c-M y c$ as a potential actor in this process. Interestingly, a similar phenotype (decrease in late replication patterns and c-Myc up-regulation) was also observed in the ventricular zone of the neural tube where Yap is expressed, suggesting that YAP function in S-phase progression may also hold true in other neural precursor cells (Figure 6A-D).

\section{Yap loss of function induces DNA damage}

DNA replication stress results in DNA damage and consequent genomic instability (Zeman and Cimprich, 2014). We thus examined the expression of phosphorylated histone $\mathrm{H} 2 \mathrm{AX}(\gamma-\mathrm{H} 2 \mathrm{AX})$, the most sensitive marker for DNA double-strand breaks (Rogakou et al., 1998). The number of $\gamma$-H2AX-positive cells was significantly increased in Yap-MO-injected CMZ compared to controls (Figure 7A,B). Since extensive DNA damage may trigger apoptosis, we next turned to a TUNEL assay and found that cell death was indeed severely increased in morphant retinas (Figure 7C,D). Surprisingly, the majority of apoptotic cells was found at 'the exit' of the CMZ close to the neural retina, and not in its most peripheral part where Yap is expressed. This strongly suggests that apoptosis occurs as a secondary consequence in late progenitor cells generated from stem cells that experienced YAP function inhibition. 
A
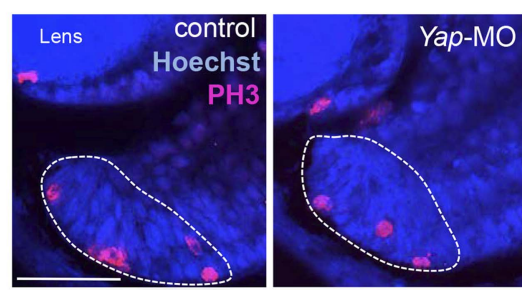

C

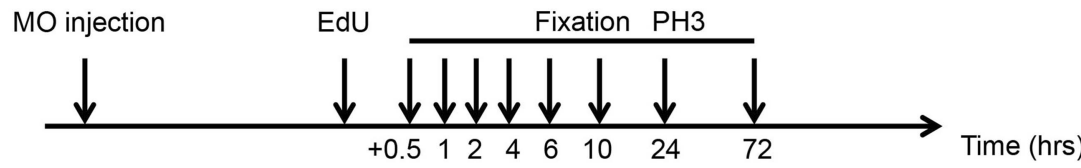

D
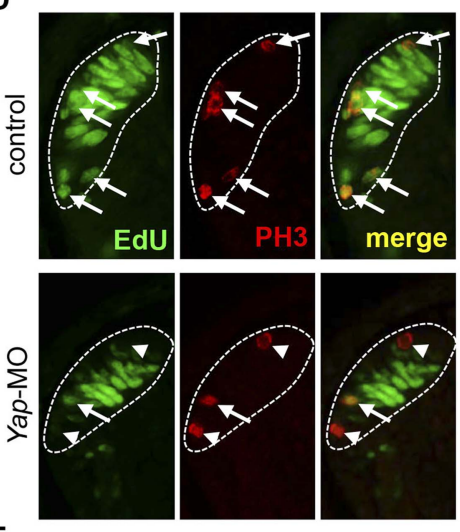

F

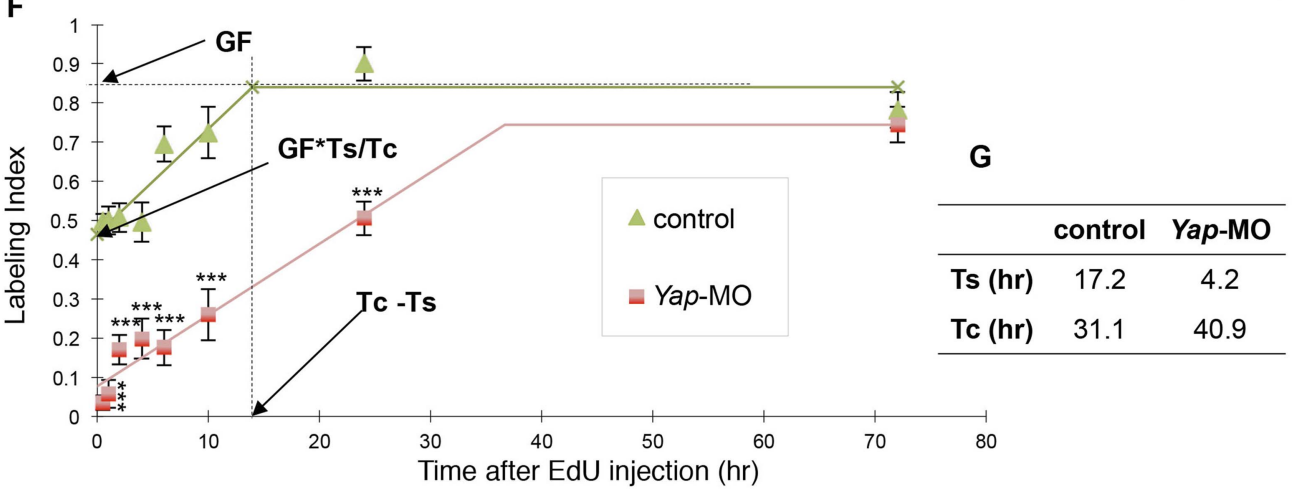

B

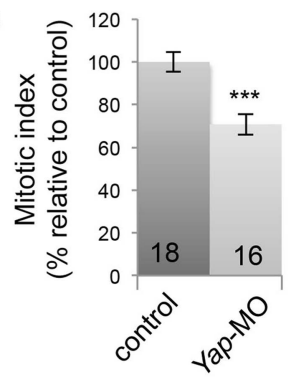

E

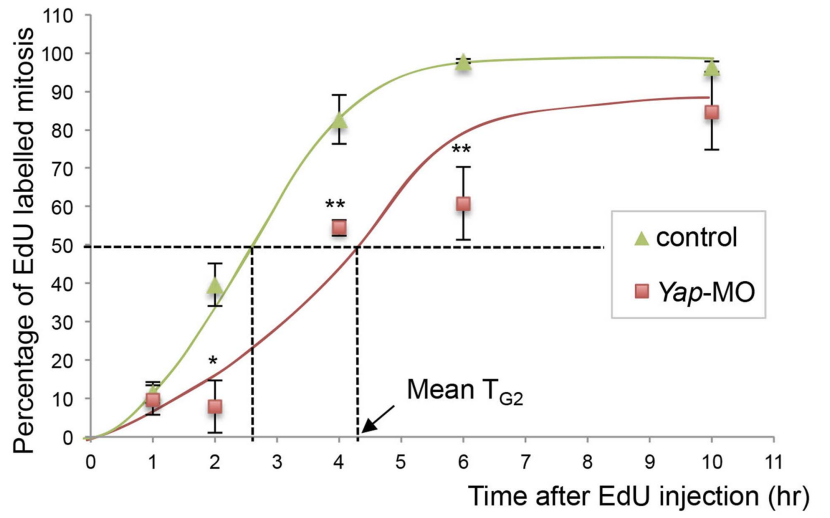


A

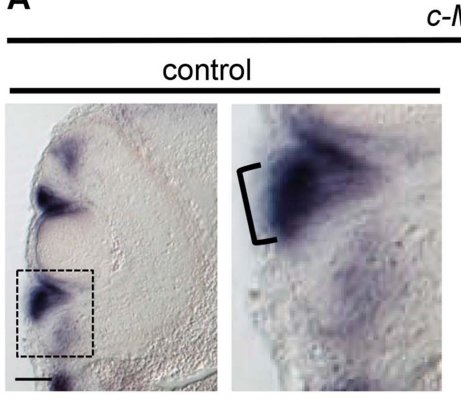

C

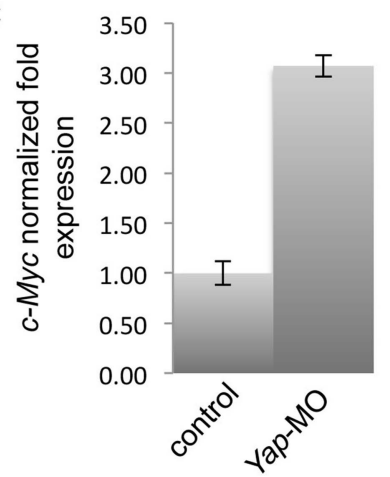

E
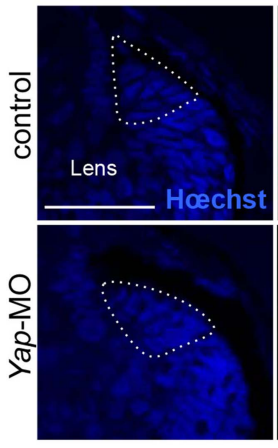

$c-M y c$

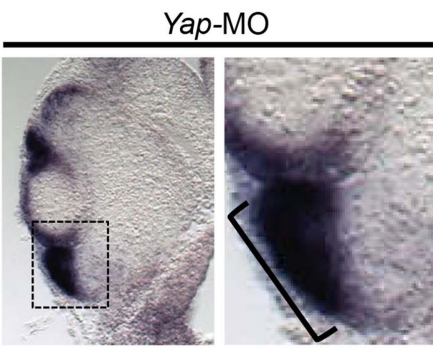

D

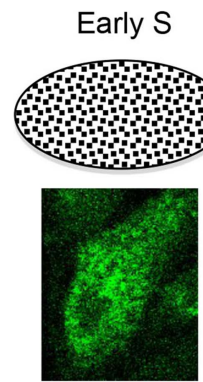

Early $S$
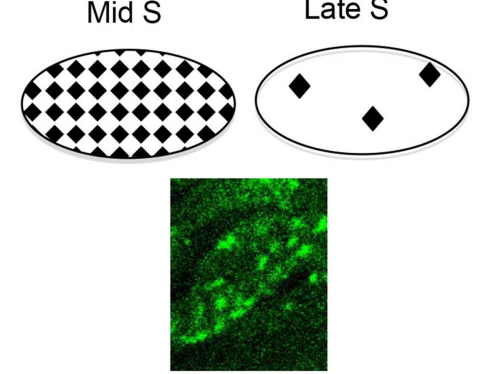

$\mathbf{F}$
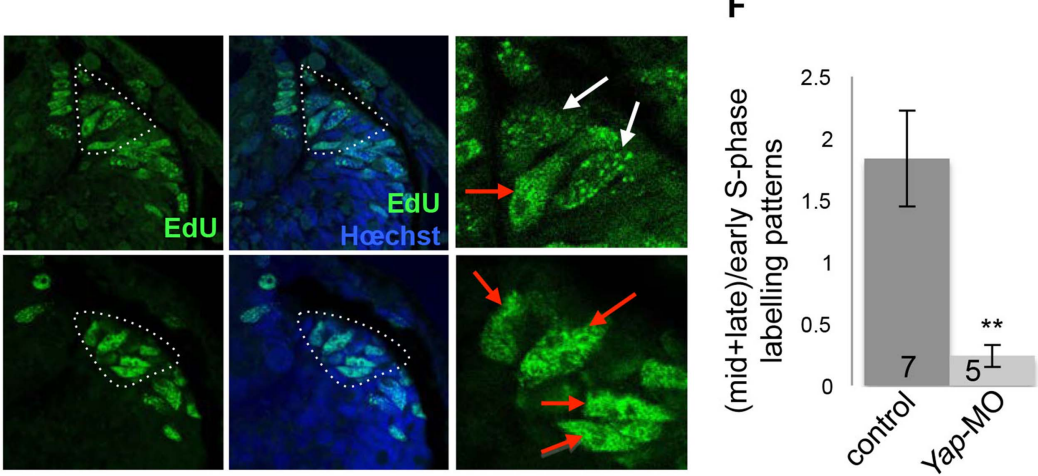

B

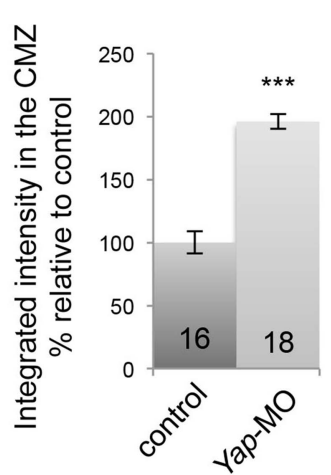

Figure 5. Yap loss of function affects the temporal program of retinal stem cell DNA replication. (A) In situ hybridization analysis of c-Myc expression on stage 40 retinal sections following two-cell stage microinjection of Yap5-mismatch-MO (control) or Yap-MO. Images on the right show higher magnifications of the CMZ (dotted lines). Note the strong expansion of c-Myc expression area (bracket). (B) Quantification of the staining in the CMZ. The number of analyzed retinal sections per condition is indicated in each bar. (C) qPCR analysis of c-Myc expression in the retina of tadpoles injected as in (A). (D) Schematic representation of replication foci observed during S-phase progression, as inferred from EdU labeling. Pictures illustrate two examples of EdU-labeled foci observed in control CMZ cells, one homogenous (early S-phase) and one with large dots (mid/late S-phase). (E) Analysis of EdU-labeled replication foci (1 hr-pulse) in the CMZ of stage 40 tadpoles injected as in (A). Enlargements of the CMZ tip (dotted lines) are shown on the right. Early (red arrows) and mid/late profiles (white arrows) were distinguished.

(F) Corresponding quantification. The number of analyzed retinas per condition is indicated in each bar. Data are represented as mean \pm SEM. Scale bar $=40 \mu \mathrm{m}$.

DOI: 10.7554/eLife.08488.012

contribute to the reduced eye size, proliferating $\mathrm{CMZ}$ cells were pulse-labeled and their progeny chased and counted in the three retinal layers (Figure 7E). As expected, we found that fewer neurons were generated in a 2-day period in Yap morphant retinas compared to control ones (Figure 7F). 


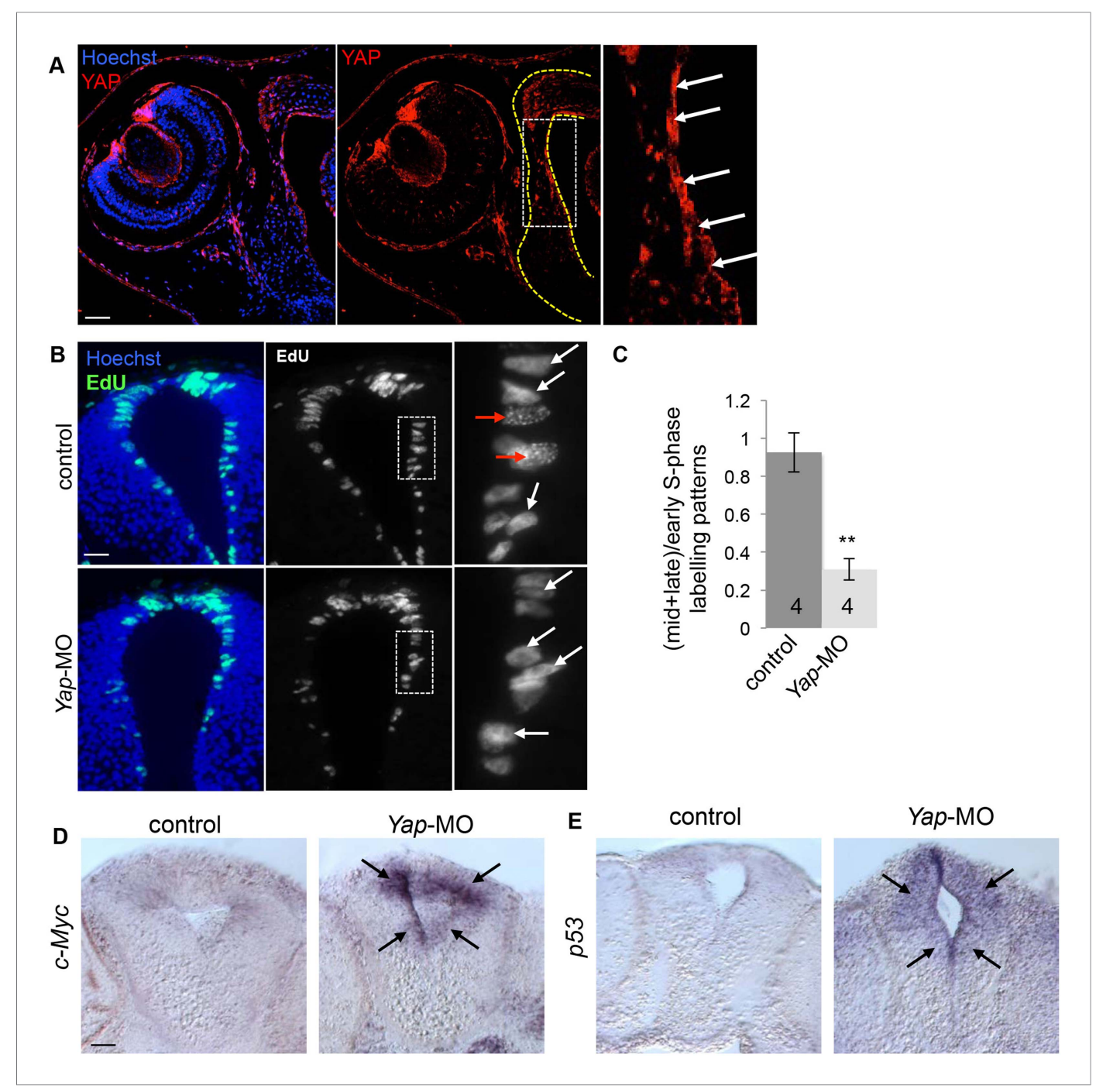

Figure 6. Effects of Yap knockdown in the neural tube. (A) Immunostaining with anti-YAP antibody on stage 42 sections. The left side of the neural tube is delineated with yellow dotted line. A higher magnification of the ventricular zone (white dotted line) is provided in the right panel. YAP labeling is most strongly detected in this region where progenitor cells reside (arrows). (B) Analysis of EdU-labeled replication foci (1 hr-pulse) in the neural tube of stage 40 tadpoles following two-cell stage microinjection of Yap-5-mismatch-MO (control) or Yap-MO. Enlargements (dotted lines) are shown on the right. Early (red arrows) and mid/late profiles (white arrows) were distinguished. (C) Corresponding quantification. The number of analyzed tadpoles per condition is indicated in each bar. Data are represented as mean \pm SEM. (D, E) In situ hybridization analysis of c-Myc or p53 expression in the neural tube of stage 40 tadpoles injected as in (B). Note the strong upregulation in the ventricular zone of the neural tube (black arrows). Scale bars $=40 \mu \mathrm{m}$.

DOI: 10.7554/eLife.08488.013

\section{Yap knock-down in the CMZ activates the p53-p21 pathway}

In order to gain additional insight into the molecular mechanisms underlying the Yap knockdown phenotype, we analyzed the expression of 15 genes encoding cell cycle regulators using the NanoString technology. Among them, only p53 and p21 cip1/WAF1 (previously named Xic2 in Xenopus and p21 hereafter) were significantly affected, with much higher levels present in Yap morphant retinas compared to control ones (Figure 7G). The tumor suppressor protein p53 is activated in response to a variety of cellular stresses (including DNA damage) and triggers cell cycle arrest or apoptosis. In situ hybridization analysis revealed that its expression in the wild type retina is restricted to the CMZ. In addition and consistent with the NanoString data, p53 staining was strongly enhanced in the CMZ of Yap-MO-injected tadpoles (Figure $7 \mathrm{H}$ ). Of note, an up-regulation was 


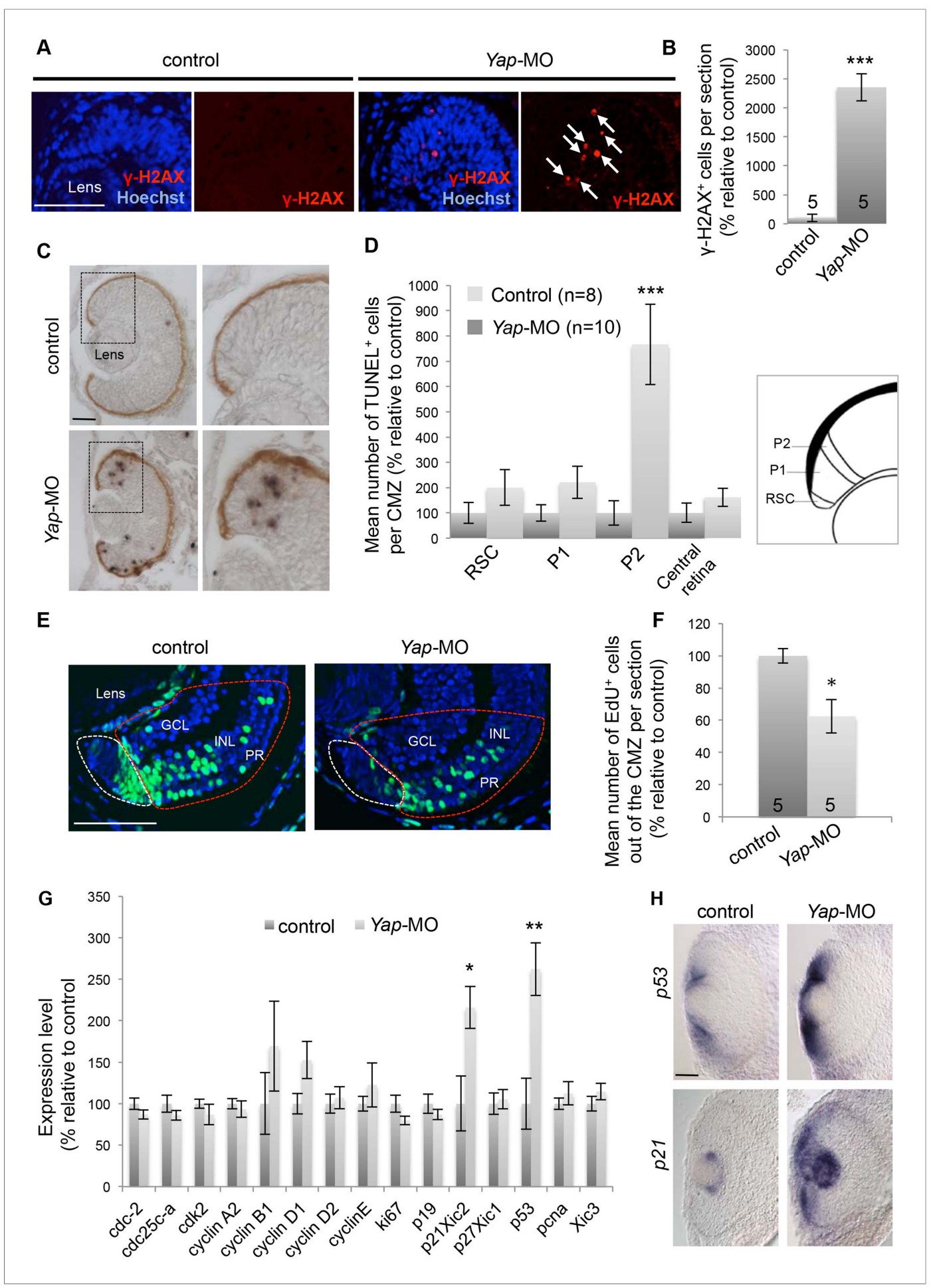

Figure 7. Yap loss of function induces DNA damage. (A) $\gamma-\mathrm{H} 2 \mathrm{AX}$ immunolabeling in the $\mathrm{CMZ}$ of retinal sections from stage 40 tadpoles following two-cell stage microinjection of Yap-5-mismatch-MO (control) or Yap-MO. Arrows point to $\gamma$-H2AX-positive cells. (B) Corresponding quantification. (C) TUNEL assay on retinal sections from stage 40 tadpoles injected as in (A). Images on the right show higher magnifications of the CMZ delineated with dotted lines. (D) Quantification of TUNEL-positive cells in the different compartments of the CMZ as illustrated on the schematic. (E) 2 days-chase of EdU-labeled cells in the CMZ of stage 42 tadpoles injected as in (A). EdU-positive cells inside the zone encircled with a red dotted line have exited the CMZ (white dotted lines) and integrated the different retinal layers. GCL: ganglion cell layer; INL: inner nuclear layer; PR: photoreceptor layer. (F) Quantification of Figure 7. continued on next page 
Figure 7. Continued

EdU-positive cells in the neural retina layers. (G) mRNA expression levels of cell cycle genes as measured with the NanoString nCounter system in heads from stage 40 tadpoles following two-cell stage microinjection of Standard MO (control) or Yap-MO. Data are the mean of four independent experiments. (H) In situ hybridization analyses of p53 and p21 expression on retinal sections from stage 40 tadpoles injected as in (A). The number of analyzed retinas per condition is indicated in each bar $(\mathbf{B}, \mathbf{F})$ or on the graph $(n$ in $\mathbf{D})$. Data are represented as mean \pm SEM. Scale bar $=40 \mu \mathrm{m}$.

DOI: 10.7554/eLife.08488.014

observed in the neural tube as well (Figure 6E). p21 is a member of the CIP/KIP family of cyclindependent kinase inhibitors that blocks the G1-S transition and has emerged as a key player in the p53 pathway (Attardi and DePinho, 2004). Since it was previously described as a lens-specific marker in the Xenopus eye (Daniels et al., 2004), we asked if it could be linked with the observed CMZ phenotype. We thus performed in situ hybridization and observed a strong ectopic p21 labeling within the $\mathrm{CMZ}$ of Yap morphant tadpoles (Figure $7 \mathrm{H}$ ). As a whole, these results suggest that increased apoptosis and probably delayed cell cycle progression resulting from Yap knockdown might be driven by the p53-p21 pathway.

\section{PKNOX1 is a novel partner of YAP in the retina}

As stated above, YAP is a co-transcriptional activator that functions in association with transcription factors such as its classical partners of the TEAD family. Among other interacting factors described so far is Drosophila Homothorax (Peng et alo, 2009; Zhang et alo, 2011). Interestingly, PKNOX1 (also named PREP1), a mammalian Homothorax ortholog belonging to the Meis/Prep homeodomain factor family, has recently been involved in the maintenance of genomic stability (lotti et alo, 2011). However, its physical interaction with YAP has not yet been reported in vertebrates. We found that the two proteins indeed interact in vitro using a two-hybrid assay (data not shown). In order to address whether they might do so in vivo as well, we performed a bimolecular fluorescence complementation (BiFC) experiment (Figure 8A) (Ohashi and Mizuno, 2014). In this purpose, constructs encoding YAP, PKNOX1 or TEAD1 (as a positive control for interaction with YAP) fused to either the amino or carboxylterminal fragment of the VENUS fluorescent protein were transfected in HEK293T cells (Figure 8B and data not shown for inverse VN/VC fusion combinations). As expected, co-transfection of Yap and Tead1 constructs resulted in a significant nuclear BiFC signal. This was lost using a Yap ${ }^{\triangle T B S}$ mutant devoid of TEAD binding site, validating the specificity of the BiFC staining. Co-transfection of both Yap and pknox 1 constructs led to a positive BiFC signal that mainly localized to the cytoplasm. YAP/PKNOX1 interaction was further confirmed by co-immunoprecipitation analyses following expression of tagged proteins (Figure 8C).

Since we found that pknox 1 is expressed in the CMZ (Figure 9A), we next sought for potential functional interaction with YAP in retinal stem cells. A loss of function approach was first undertaken to compare Yap and pknox1 knockdown phenotypes (Figure 9B-G). The injected dose of pknox1-MO was adapted to avoid broad developmental defects (see Figure 9-figure supplement 1 for validation of pknox1-MO specificity and efficiency). Although the eye phenotype appeared more dramatic than that observed upon Yap knockdown (layering defects of the retina), it similarly led to a significant reduction in total eye size (Figure 9B,C), associated with decreased EdU incorporation in the CMZ compared to controls (Figure $9 G, H$ ). In addition, analysis of EdU-labeled replication foci revealed a decreased proportion of stem cells in mid/late S-phase (Figure 9D-E) in pknox1 morphant, as observed following Yap knockdown. These embryos also displayed upregulation of c-Myc expression in the $\mathrm{CMZ}$, thus recapitulating main features of the Yap knockdown phenotype (Figure 9F). We then asked whether YAP and PKNOX1 might synergize in a co-overexpression assay. Of note, pknox1 overexpression alone does not significantly affect CMZ cell proliferation. The injected dose of Yap mRNA was lowered so that it only leads to a moderate although significant increase in the number of EdU-labeled cells. In these conditions, pknox 1 mRNA injection was found to exacerbate Yap gain of function phenotype (Figure 9, J). Finally, we tested whether pknox 1 knockdown might rescue the overproliferative effects of Yap misexpression. We found indeed that EdU incorporation was restored to a basal level in a pknox1 morphant context (Figure 9K,L). Together, these data support the idea that PKNOX1 physically and functionally interacts with YAP in the CMZ. 


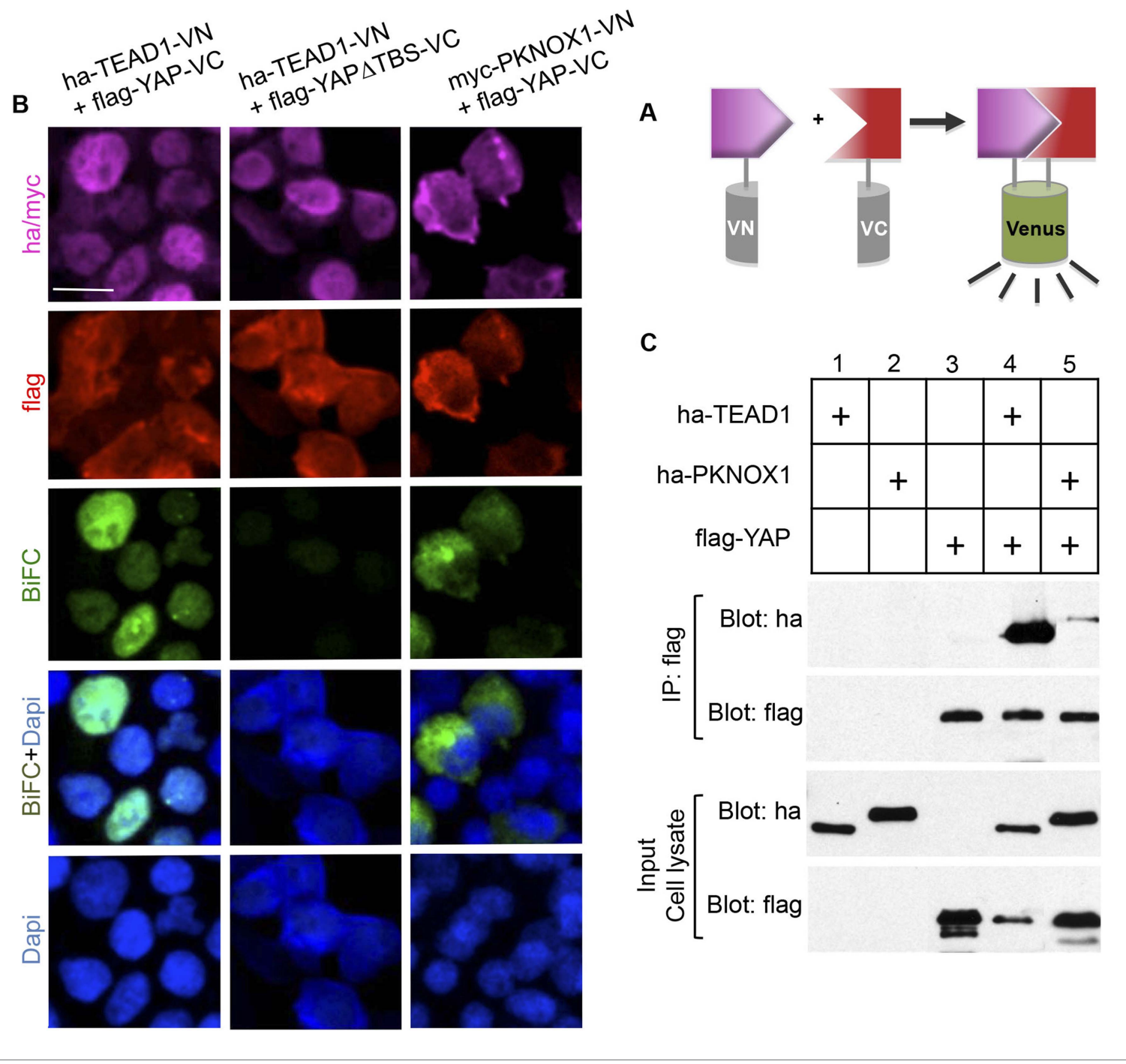

Figure 8. Physical interaction between YAP and PKNOX1. (A) Schematic representation of BiFC principle. (B) Immunolabeling/BiFC analysis on HEK293T cells transfected with VN and VC chimeric constructs, as indicated. (C) Co-immunoprecipitation assay of 293T cells transfected with tagged constructs as indicated. Scale bars $=20 \mu \mathrm{m}$.

DOI: 10.7554/eLife.08488.015

\section{Discussion}

Long-term maintenance of tissue homeostasis relies on the fine-tuning of adult stem cell activity. Our knowledge regarding the molecular basis sustaining somatic stemness features is still very limited but may have important implications for regenerative medicine and cancer therapy. In the present study, we identified YAP, a downstream effector of the Hippo pathway, as a stem cell specific marker required for homeostatic growth of the frog post-embryonic retina. Our in vivo loss of function approach unexpectedly revealed a novel role for YAP in governing DNA replication timing of retinal stem cells. We propose a model in which this function would contribute to the maintenance of their genomic stability (Figure 10). Importantly, based on our findings in the neural tube, we propose that such function might be generalizable to other neural precursor populations. Whether this involves YAP cytoplasmic and/or nuclear activity remains an open question.

The ability of nuclear YAP to expand stem/progenitor cell populations has been established in numerous model systems (Barry and Camargo, 2013; Piccolo et al., 2014). However, the mechanisms underlying altered cell proliferation (changes in cell cycle length and/or re-entry) are rarely investigated 

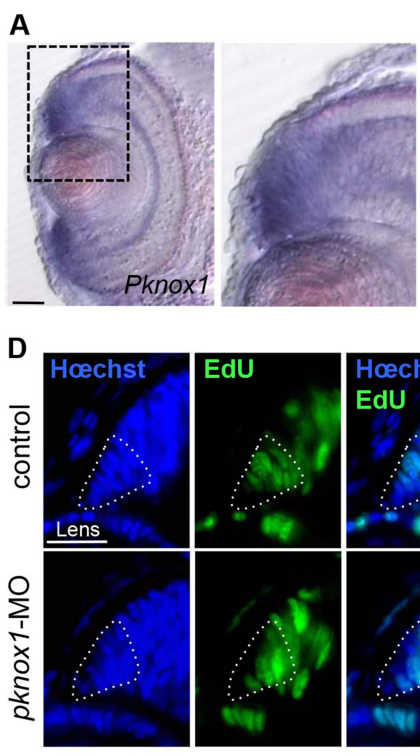

G
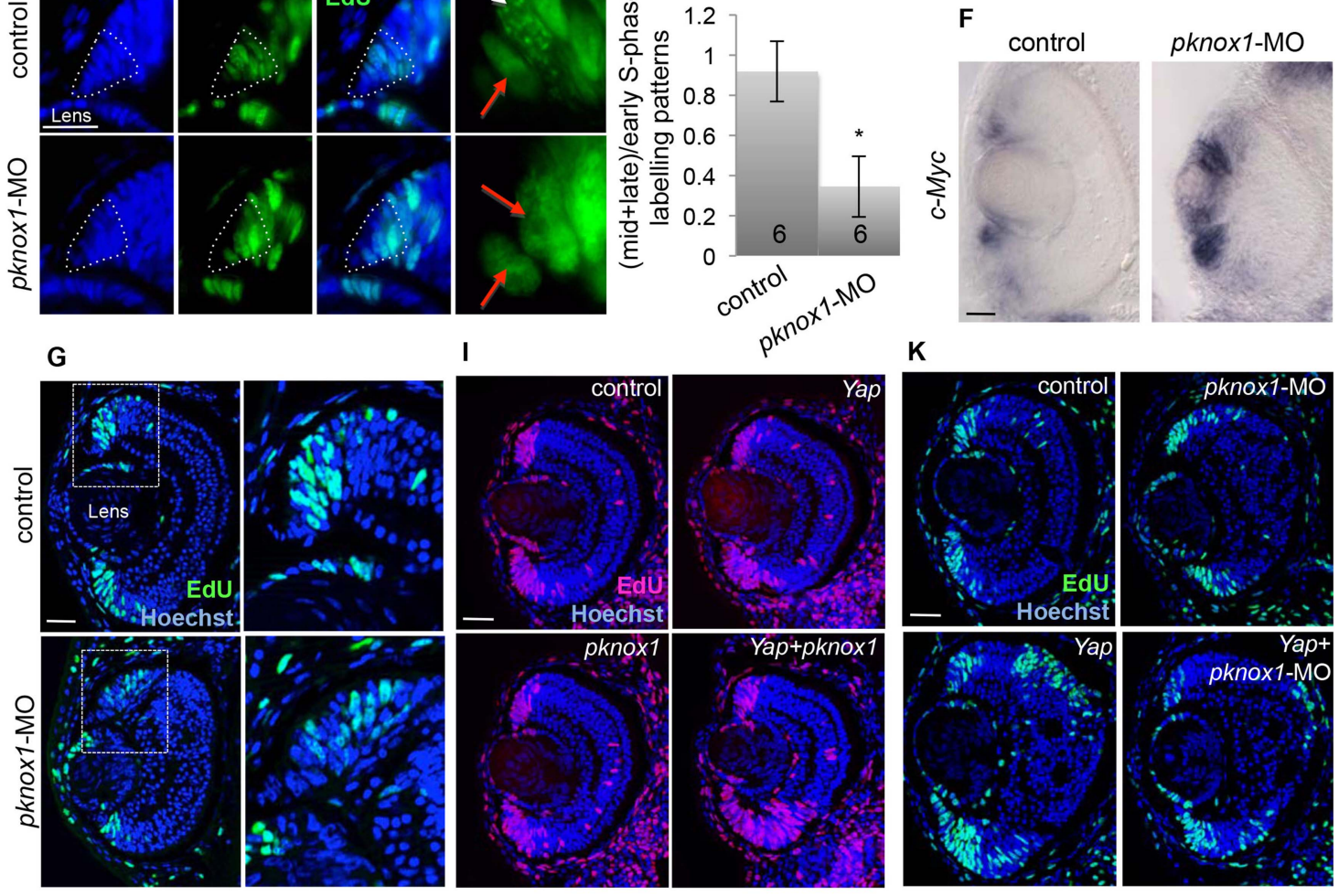

K
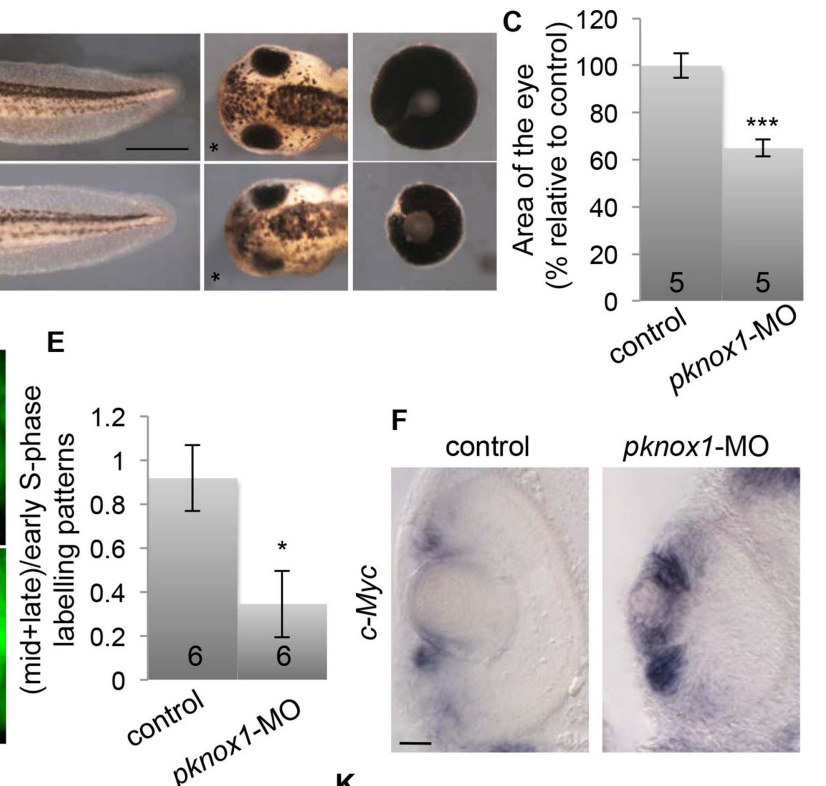

.

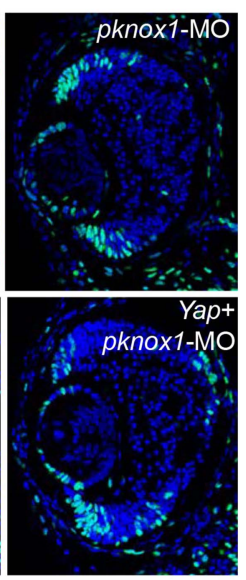

H
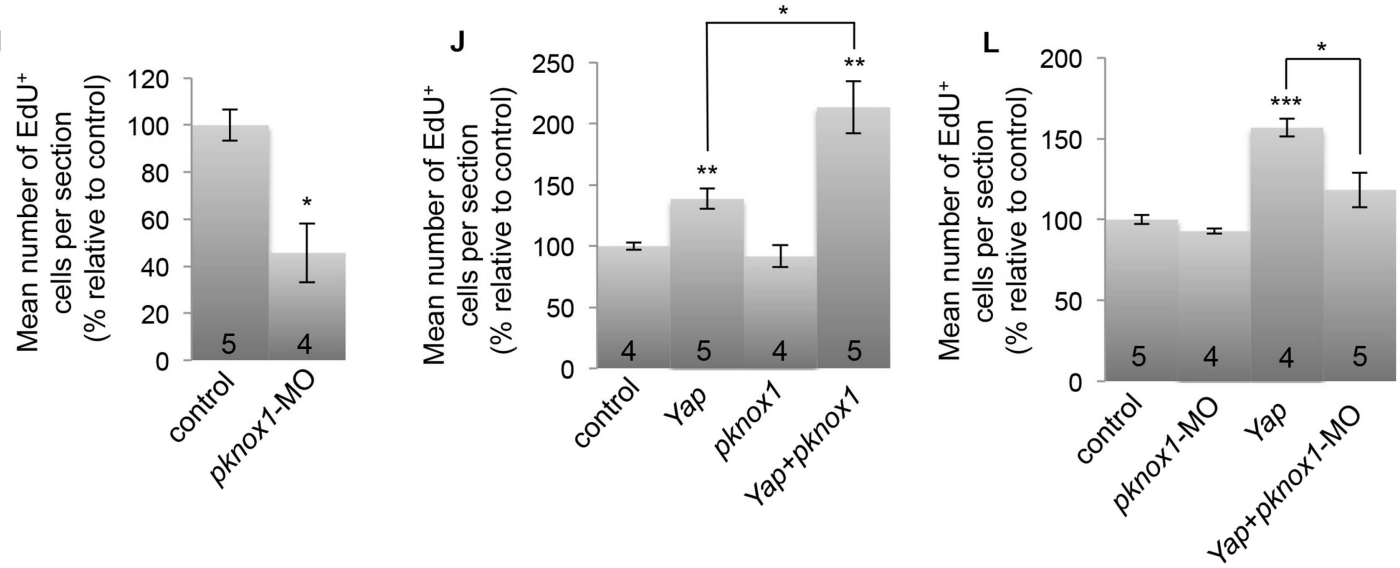

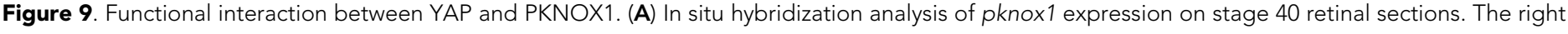

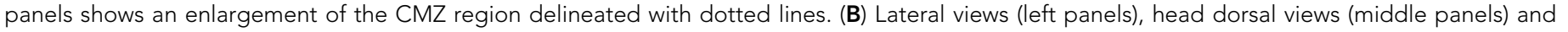

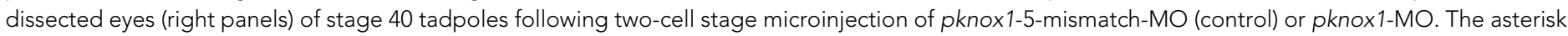

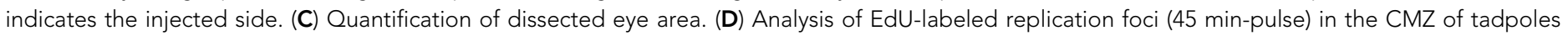
injected as in (B). Enlargements of the CMZ tip (dotted lines) are shown on the right. Early (red arrows) and mid/late profiles (white arrows)

Figure 9. continued on next page 
Figure 9. Continued

were distinguished. (E) Corresponding quantification. (F) In situ hybridization analysis of c-Myc expression on stage 40 retinal sections from tadpoles injected as in (B). (G-L) EdU incorporation assays (3-hr pulse) analyzed on retinal sections from stage 40 tadpoles. (G, H) shows the effect of pknox1 knockdown (injection of pknox1-5-mismatch-MO (control) or pknox1-MO). (I, J) shows the synergistic effects of pknox1 and Yap (injection of GFP mRNA and either B-gal mRNA (control), Yap + B-gal mRNA (Yap), pknox1 + B-gal mRNA (pknox1), Yap + pknox1 mRNA (Yap + pknox1)). (K, L) shows the rescue of Yap overexpression by pknox1 knockdown (injection of either pknox1-5-mismatch-MO + B-gal mRNA (control), pknox1-MO + B-gal mRNA (pknox1-MO), pknox1-5-mismatch-MO + Yap mRNA (Yap), pknox1-MO + Yap mRNA (Yap + pknox-MO)). Of note, a suboptimal dose of pknox1-MO was used for the rescue experiment so that it does not alone give any eye phenotype. The total number of analyzed retinas per condition is indicated in each bar. Scale bar $=1 \mathrm{~mm}$ in (B) and $40 \mu \mathrm{m}$ for all other panels.

DOI: 10.7554/eLife.08488.016

The following figure supplement is available for figure 9:

Figure supplement 1. Validation of pknox1-MO efficiency and specificity.

DOI: 10.7554/eLife.08488.017

in detail. In the developing neural tube for instance, YAP-driven increase in neural progenitor cell number has been proposed to result from accelerated cell cycle progression but whether its loss of function alters cell cycle kinetics as well remains an open question (Cao et al., 2008; Zhang et al., 2012). As observed in other adult organs in mammals (Azzolin et alo, 2014; Chen et al., 2014; Zhang et al., 2014), our results suggest that YAP is dispensable for the maintenance of the stem cell pool. We instead uncovered that its depletion lengthens retinal stem cell divisions. However, to our surprise, this is associated with a dramatic shortening of their S-phase. It should be emphasized that such phenotype likely corresponds to a hypomorphic one due to the use of Morpholinos that allows partial loss of function. DNA replication is a tightly regulated process that follows a strict temporal program. Our observation that Yap knockdown results in a mark reduction of late S-phase labeling patterns can suggest that some firing origins have advanced their activation timing, which may account for the reduced S-phase duration. The genetic control of DNA replication temporal progression has not been elucidated yet and there are thus very few examples in the literature where gene perturbation leads to

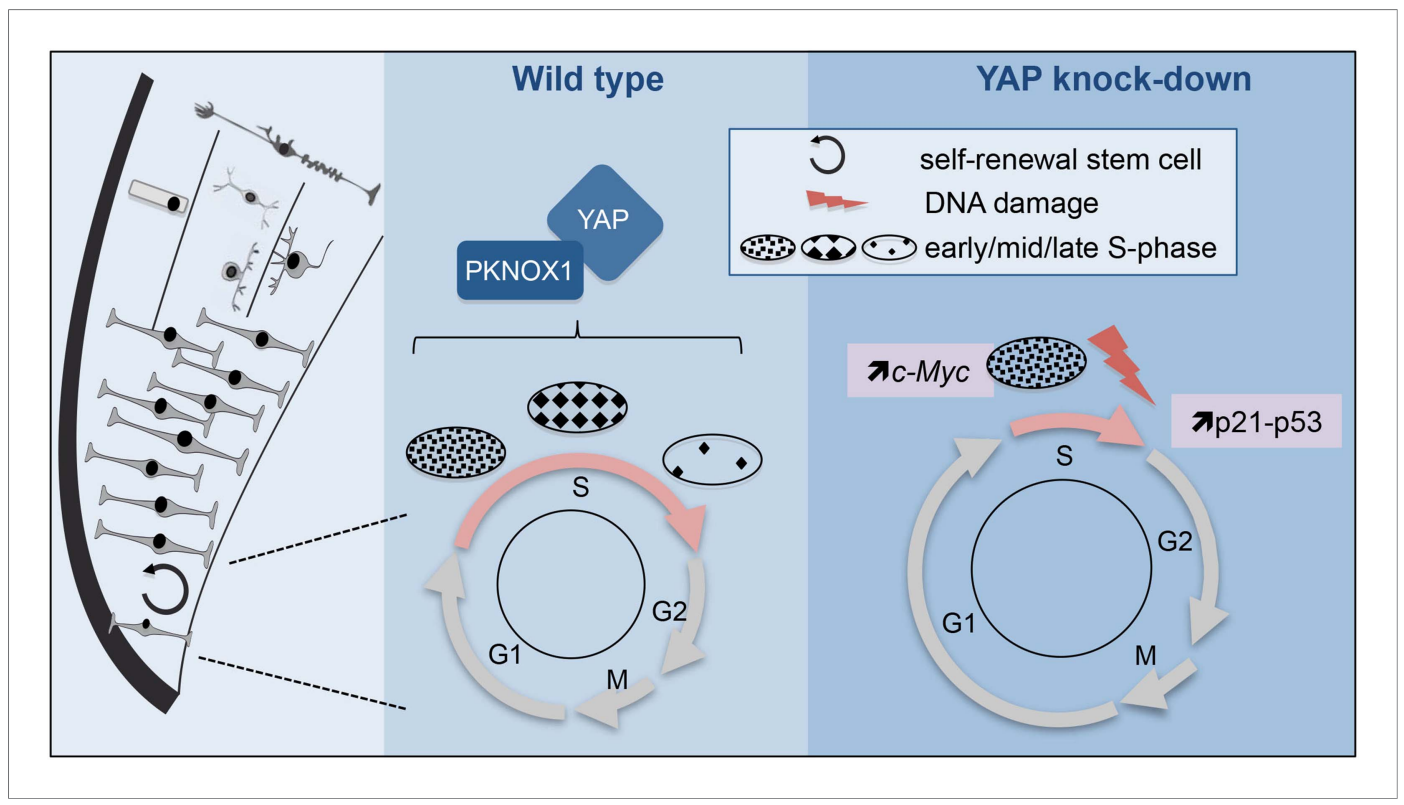

Figure 10. Model illustrating YAP function in retinal stem cells. We found that YAP is expressed in CMZ retinal stem cells (left panel). The middle panel shows the cell cycle of wild type retinal stem cells and the putative role of the YAP/PKNOX1 complex in the control of S-phase temporal progression (represented by the distinct patterns of DNA replication foci). YAP knock-down (right panel) leads to a dramatic reduction of S-phase length likely due to c-Myc-dependent premature firing of late replication origins. This results in increased occurrence of DNA damage, enhanced p21 and p53 expression and eventually cell death.

DOI: 10.7554/eLife.08488.018 
a deregulation of S-phase duration (Aparicio, 2013; Yamazaki et al., 2013). Beyond its wellcharacterized transcriptional activity, it was reported that c-Myc exerts a non-transcriptional control on DNA replication. Its overexpression indeed causes increased replication origin activity and consequent S-phase shortening (Dominguez-Sola et al., 2007; Robinson et al., 2009; Srinivasan et al., 2013). Intriguingly, in contrast to Drosophila or cancer cells, where c-Myc has been reported to be positively regulated by Yap (Neto-Silva et al., 2010; Stocker, 2011; Xiao et al., 2013), we found that its expression is enhanced in the CMZ of Yap morphant tadpoles. Although the underlying mechanism deserves further investigation, this raises the hypothesis that c-Myc may contribute to the S-phase defects caused by Yap knockdown. Besides, in addition to be involved in replication progression, we do not exclude that YAP may also regulate replication origin licencing in G1, as recently reported in human umbilical vein endothelial cells (Shen and Stanger, 2015). This might explain the observed lengthening of the cell cycle as a result of impaired G1/S transition. Alternatively, delayed G-phase progression might occur as a secondary consequence of S-phase defects.

Replication stress is a source of DNA damage, which may ultimately trigger activation of the p53-p21 pathway (Bartek and Lukas, 2001). As observed following c-Myc overexpression in vitro (Dominguez-Sola et al., 2007; Robinson et al., 2009; Srinivasan et al., 2013), we found an increased occurrence of double-strand breaks in Yap morphant retina, associated with an upregulation of both $p 53$ and p21. Since p21 is known to inhibit G1/S and G2/M transitions and p53 to induce programmed cell death (Vogelstein et al., 2000), this could contribute to both the lengthening in $\mathrm{G}$ phases and the increased number of apoptotic cells.

These findings raise key questions regarding specific features of stem cell biology. In addition to unique properties (such as the ability to self renew), emerging evidence suggests that somatic stem cells also differ from progenitor cells in the way they regulate basic cellular processes including their metabolic state (Burgess et al., 2014) or DNA-damage responses (Insinga et al., 2014). Regarding cell cycle progression, it has been shown during development that mammalian cortical neural stem cells exhibit a substantially longer S-phase than progenitors committed to neuron production (Arai et al., 2011; Turrero García et al., 2015). It was thus proposed that neural stem cells may need to invest more time during S-phase into quality control of replicated DNA. In agreement with this, we also found that CMZ retinal stem cells exhibit a longer S-phase compared to fast amplifying progenitors (data not shown). In addition, our work points for the first time towards a factor, YAP, that may be critically involved in this stem cell specific regulation of S-phase. Although its precise function in this process remains to be investigated, it indeed appears to be required for proper choreography of the DNA replication program and may as such be necessary to maintain genomic integrity of retinal stem cells.

Our findings also have important medical implications since aberrant DNA replication timing has been proposed to be a causative factor in diseases such as cancer and neuronal disorders (Aladjem, 2007; Watanabe and Maekawa, 2010; Donley and Thayer, 2013). Interestingly, another component of the Hippo pathway, LATS1, has very recently been involved in ATR-mediated response to replication stress in lung cancer cells (Pefani et al., 2014). Several Hippo pathway components may thus regulate (independently or in concert) S-phase progression and quality control and thereby safeguard genomic integrity.

Although Homothorax is known to partner the Drosophila Yap homologue Yorkie in some developmental contexts (Peng et al., 2009; Zhang et al., 2011), this has not been reported yet for its vertebrate orthologs. Here, we provide biochemical and functional evidences supporting an interaction between PKNOX1 and YAP in the retina. PKNOX1 belongs to the TALE (three amino acids loop extension) class of homeodomain proteins and is involved in many developmental processes (Berthelsen et al., 1998; Ferretti et al., 2006). Down-regulation of pknox1 in both zebrafish and mouse embryos leads to a small eye phenotype (Deflorian et al., 2004; Ferretti et al., 2006), reminiscent of what we found in Xenopus. Interestingly, pknox 1 inactivation was reported to trigger cell death in the zebrafish CNS (Deflorian et al., 2004). Furthermore, pknox1 deficiency leads to increased DNA damage and apoptosis both in embryonic fibroblasts and in the mouse epiblast (Micali et al., 2009; Fernandez-Diaz et al., 2010; lotti et al., 2011). On the basis of these different reports and our findings, we thus propose that PKNOX1 and YAP interact together to maintain genomic stability in retinal stem cells. Whether PKNOX1 functions in competition with TEAD for YAP interaction or whether they all associate in a tripartite complex are important questions to be addressed in the future. 


\section{Materials and methods}

\section{Experimental procedures}

\section{Ethics statement}

All animal care and experimentation were conducted in accordance with institutional guidelines, under the institutional license C 91-471-102. The study protocol was approved by the institutional animal care committee: the Direction Départementale de la Protection des Populations.

Plasmids and morpholinos

HA-tagged Xenopus constructs encoding wild-type or mutant YAP proteins (non-phosphorylable YAP ${ }^{S 98 A}$ or TEAD-binding site-deleted $Y^{A}{ }^{\triangle T B S}$ ) were provided by $S$ Gee and S Moody (Gee et al., 2011) and subcloned into pCS2+. The Tead1 ORF, a gift from P Thiébaud (Naye et al., 2007), was subcloned into pCS2+Flag. The pknox1 (prep1, NM_001096382) full-length cDNA sequence was cloned by RT-PCR into pCS2+Flag. Yap, pknox1 and Tead1 ORF were cloned in frame upstream the non-fluorescent N-ter or C-ter fragments of VENUS fluorescent protein (a gift from J Smith) in HA-, myc- or flag-tagged pCS2+ (Saka et al., 2008). PCR primer sequences are listed in Supplementary file 1. Translation-blocking antisense Morpholino oligonucleotides (MO, GeneTools, Philomath, OR, United States) and PhotoMorpholinos (Photo-MO, GeneTools) used in this study are also listed in Supplementary file 1.

\section{Microinjection}

200 pg of mRNA (synthesized with mMessage mMachine kit, Life Technologies, Carlsbad, CA, United States) or $2 \mathrm{pmol} \mathrm{MO}$ were injected in one or two blastomeres at the two-cell stage. mRNAs encoding ß-Galactosidase or GFP were injected as controls and/or lineage tracers. In some experiments, the injected side was identified using the fluorescence of lissamine tagged-MO. Cleavage of antisense or sense Photo-MO was performed by exposing embryos/tadpoles to UV light (365 nm) for $10 \mathrm{~min}$ in a glass surrounded by aluminum. A ratio of 0.9:1 Yap-S-photo-MO to Yap-MO was used.

EdU labeling, immunostaining and TUNEL assay

Tadpoles were injected intra-abdominally with $1 \mathrm{mM}$ of 5-ethynyl-20-deoxyuridine (EdU, Invitrogen, Carlsbad, CA, United States) and fixed at the required stage in $4 \%$ paraformaldehyde. For cumulative labeling experiments, EdU was made constantly available for the desired time-period following injection by incubating tadpoles in a $1 \mathrm{mM}$ EdU solution, renewed every other day. EdU incorporation was detected on paraffin sections using the Click-iT EdU Imaging Kit according to manufacturer's recommendations (Invitrogen). Immunostaining was performed following 4\% paraformaldehyde fixation on paraffin sections with antibodies listed in Supplementary file 2. Standard procedures were used unless specified. Detection of apoptotic cells was carried out with the DeadEnd fluorometric TUNEL system (Promega, Fitchburg, WI, United States) or using TdT-driven dig-dUTP incorporation (Roche, Basel, Switzerland) followed by immunolabeling and NBT/BCIP staining, according to the manufacturer's instructions. Cell nuclei were stained with Hoechst (Sigma-Aldrich, St. Louis, MI, United States) or DAPI (Thermo scientific, Waltham, MA, United States).

\section{Analysis of cell-cycle parameters}

For cumulative EdU incorporation assay in retinal stem cells, EdU-labeled and -unlabeled nuclei were scored among the 3 most peripheral CMZ cells on retinal sections (3-8 retinas analyzed per condition). The mean labeling index (LI) per retina was then plotted as a function of time after EdU injection. $\mathrm{LI}$ increases linearly until it reaches a plateau ( $T_{\text {plateau }}$ ) allowing determining the growth fraction (GF; proportion of cycling cells within the considered population). The best-fit line was drawn using the Excel spreadsheet provided by $\mathrm{R}$ Nowakowski (Nowakowski et al., 1989). It allows estimating $T_{c}$ and $T_{S}$ using the two following equations: $T_{\text {plateau }}=T_{c}-T_{s} ; L I_{0}=G F \times\left(T_{s} / T_{c}\right)\left(L I_{0}\right.$ being the extrapolated $y$ intercept of the best-fit line). Mitotic index and percentages of EdU-labeled mitosis were measured as previously described (Locker et al., 2006). The time required for half-maximal appearance of EdU labeling in the mitotic population was taken as an estimation of the mean G2 length $\left(T_{G 2}\right.$ ) (Arai et al., 2011).

In situ hybridization

Digoxigenin-labeled antisense RNA probes were generated according to the manufacturer's instruction (Roche) from the following PBS plasmids: cMyc, a gift from WA Harris (Xue and Harris, 
2011); p21, a gift from S Ohnuma (Daniels et al., 2004); Yap, a gift from S Moody (Gee et al., 2011); Taz (ImaGenes clone no. 102278); Tead1 and Tead2, a gift from P Thiébaud (Naye et al., 2007). p53 RNA probe was generated from a sequence encompassing the entire $p 53$ coding region that was amplified by RT-PCR and cloned into the pCS2+Flag vector (PCR primer sequences are listed in Supplementary file 1). Whole mount in situ hybridizations were carried out as previously described (Perron, 2003) and analyzed on $50 \mu \mathrm{m}$ vibratome sections. For double EdU/Yap mRNA staining, in situ hybridization was performed on cryostat section as previously described (Perron et al., 1998) followed by EdU detection.

\section{RNA extraction}

Total RNA from 50 tadpole heads (for subsequent NanoString experiment) or 70 dissected retinas (for subsequent qPCR) was isolated using the Trizol reagent (Life Technologies) and quality assessed using the Experion automated electrophoresis system (BioRad, Hercules, CA, United States).

\section{Quantitative real-time PCR}

Reverse transcription was performed using the iScript cDNA Synthesis Kit (BioRad). qPCR reactions were performed in triplicate using SsoFast EvaGreen Supermix (BioRad) on a C1000 thermal cycler (CFX96 real-time system, BioRad). Results were normalized against the expression of reference genes ODC and RPL8 using CFX Manager software (BioRad). PCR primer sequences are listed in Supplementary file 1.

Nanostring

The nCounter Analysis System (NanoString Technologies, Seattle, WA, United States) was used according to the manufacturer's instructions with $1000 \mathrm{ng}$ of total RNA to profile 15 cell cycle genes for which we designed custom CodeSets (see Supplementary file 3). The readouts represent counts of individual fluorescent barcodes and provide a sensitive measurement of the selected RNA expression levels in the sample. The Nanostring data were analyzed using the nSolver Analysis Software 2. Background subtraction was performed by substracting the average plus two times the standard deviation of the 8 negative internal controls. Data were normalized as a geometric mean, against the internal positive control spikes and afterwards against three housekeeping genes (see Supplementary file 3).

\section{Co-immunoprecipitation assay and Western blot}

Western blot were conducted using standard procedures on Xenopus embryo/tadpole protein extracts. Immunoprecipitation assays on HEK293T protein extracts were performed with the Dynabeads Protein A Immunoprecipitation Kit (Invitrogen) according to the manufacturer's protocol. Antibodies used are listed in Supplementary file 2.

BiFC analysis

For BiFC experiments, plasmids (50 ng each) were transiently transfected in HEK293T cells using lipofectamine 2000 reagent (Invitrogen). Cells were grown for $16 \mathrm{hr}$, fixed and immunostained using standard procedures with antibodies listed in Supplementary file 2.

\section{Microscopy}

Fluorescence and brightfield images were captured with an ApoTome-equiped Axio Imager.M2 microscope and processed using AxioVision REL 7.8 software (Zeiss, Oberkochen, Germany) or with a LSM 700 confocal microscope using ZEN software (Zeiss). Confocal pictures represent a merged zstack of 15 slices ( $1 \mu \mathrm{m}$ each).

Quantification and statistical analyses

For quantifications of labeled cells by manual cell counting in the CMZ, 6 to 10 sections per retina and a minimum of 3 retinas were analyzed. Dissected eye area, Hoechst/PCNA labeling surface or in situ hybridization staining intensity in the CMZ were measured using Adobe Photoshop CS4 software. All experiments were performed at least in duplicate. Shown in figures are results from one representative experiment unless specified. Statistical analyses were performed by Student's $t$-test. Statistical significance is: ${ }^{*} p<0.05 ;{ }^{* *} p<0.01 ;{ }^{* * *} p<0.001$; n.s. not significant.

\section{Acknowledgements}

We thank S Gee, S Moody, P Thiébaud, WA Harris, S Ohuma and J Smith for providing plasmids. We are grateful to J Hamdache, E Braginskaja, A Ravigneaux for technical assistance and A Chesneau, 
C de Medeiros and E Henry for animal care. We are indebted to K Marheineke for helpful discussions and grateful to $X$-J Yang for insightful comments on the manuscript. This research was supported by grants to MP from the ANR, Idex Paris-Saclay, Retina France, CNRS, Universite Paris-Sud, and grants to $\mathrm{KH}$ from Cluster of Excellence and DFG Research Center Nanoscale Microscopy and Molecular Physiology of the Brain.

\section{Additional information}

Funding

\begin{tabular}{|c|c|c|}
\hline Funder & Grant reference & Author \\
\hline $\begin{array}{l}\text { L' Agence Nationale de la } \\
\text { Recherche }\end{array}$ & ANR-10-BLAN-1220 & Muriel Perron \\
\hline $\begin{array}{l}\text { Campus Paris Saclay, Foundation } \\
\text { de Cooperation Scientifique }\end{array}$ & Idex Paris-Saclay & Muriel Perron \\
\hline Retina France & & Muriel Perron \\
\hline $\begin{array}{l}\text { Centre National de la Recherche } \\
\text { Scientifique (National Center for } \\
\text { Scientific Research) }\end{array}$ & & Muriel Perron \\
\hline Univ Paris Sud & & Muriel Perron \\
\hline Cluster of Excellence & Heidelberg University & Kristine A Henningfeld \\
\hline
\end{tabular}

The funders had no role in study design, data collection and interpretation, or the decision to submit the work for publication.

Author contributions

PC, GV-L, JB, ML, OB, MP, Conception and design, Acquisition of data, Analysis and interpretation of data, Drafting or revising the article; $\mathrm{KP}, \mathrm{RC}, \mathrm{CM}, \mathrm{CB}, \mathrm{MH}, \mathrm{KAH}$, Acquisition of data, Analysis and interpretation of data, Drafting or revising the article

Author ORCIDs

Guillermo Vega-Lopez, (iD http://orcid.org/0000-0002-2426-2844

Ethics

Animal experimentation: All animal care and experimentation were conducted in accordance with institutional guidelines, under the institutional license C 91-471-102. The study protocol was approved by the institutional animal care committee: the Direction Départementale de la Protection des Populations.

\section{Additional files}

Supplementary files

- Supplementary file 1. Sequences of Morpholino oligonucleotides and primers used in the study. DOI: 10.7554/eLife.08488.019

- Supplementary file 2. List of antibodies used in the study.

DOI: $10.7554 /$ eLife.08488.020

- Supplementary file 3. Sequences used in the NanoString experiment.

DOI: 10.7554/eLife.08488.021

\section{References}

Aladjem MI. 2007. Replication in context: dynamic regulation of DNA replication patterns in metazoans. Nature Reviews. Genetics 8:588-600. doi: 10.1038/nrg2143.

Aparicio OM. 2013. Location, location, location: it's all in the timing for replication origins. Genes \& Development 27:117-128. doi: 10.1101/gad.209999.112.

Arai Y, Pulvers JN, Haffner C, Schilling B, Nüsslein I, Calegari F, Huttner WB. 2011. Neural stem and progenitor cells shorten S-phase on commitment to neuron production. Nature Communications 2:154. doi: 10.1038/ ncomms 1155 . 
Attardi LD, DePinho RA. 2004. Conquering the complexity of p53. Nature Genetics 36:7-8. doi: 10.1038/ ng0104-7.

Azzolin L, Panciera T, Soligo S, Enzo E, Bicciato S, Dupont S, Bresolin S, Frasson C, Basso G, Guzzardo V, Fassina A, Cordenonsi M, Piccolo S. 2014. YAP/TAZ incorporation in the $\beta$-catenin destruction complex orchestrates the Wnt response. Cell 158:157-170. doi: 10.1016/j.cell.2014.06.013.

Barry ER, Camargo FD. 2013. The Hippo superhighway: signaling crossroads converging on the Hippo/Yap pathway in stem cells and development. Current Opinion in Cell Biology 1-7. doi: 10.1016/j.ceb.2012.12.006.

Barry ER, Morikawa T, Butler BL, Shrestha K, de la Rosa R, Yan KS, Fuchs CS, Magness ST, Smits R, Ogino S, Kuo CJ, Camargo FD. 2013. Restriction of intestinal stem cell expansion and the regenerative response by YAP. Nature 493:106-110. doi: 10.1038/nature11693.

Bartek J, Lukas J. 2001. Pathways governing G1/S transition and their response to DNA damage. FEBS Letters 490:117-122. doi: 10.1016/S0014-5793(01)02114-7.

Berthelsen J, Zappavigna V, Mavilio F, Blasi F. 1998. Prep1, a novel functional partner of Pbx proteins. The EMBO Journal 17:1423-1433. doi: 10.1093/emboj/17.5.1423.

Burgess RJ, Agathocleous M, Morrison SJ. 2014. Metabolic regulation of stem cell function. Journal of Internal Medicine 276:12-24. doi: 10.1111/joim.12247.

Cai J, Zhang N, Zheng Y, de Wilde RF, Maitra A, Pan D. 2010. The Hippo signaling pathway restricts the oncogenic potential of an intestinal regeneration program. Genes \& Development 24:2383-2388. doi: 10.1101/gad. 1978810.

Cai L, Hayes NL, Nowakowski RS. 1997. Synchrony of clonal cell proliferation and contiguity of clonally related cells: production of mosaicism in the ventricular zone of developing mouse neocortex. The Journal of Neuroscience 17: 2088-2100.

Cao X, Pfaff SL, Gage FH. 2008. YAP regulates neural progenitor cell number via the TEA domain transcription factor. Genes \& Development 22:3320-3334. doi: 10.1101/gad.1726608.

Centanin L, Hoeckendorf B, Wittbrodt J. 2011. Fate restriction and multipotency in retinal stem cells. Cell Stem Cell 9:553-562. doi: 10.1016/j.stem.2011.11.004.

Chen Q, Zhang N, Gray RS, Li H, Ewald AJ, Zahnow CA, Pan D. 2014. A temporal requirement for Hippo signaling in mammary gland differentiation, growth, and tumorigenesis. Genes \& Development 28:432-437. doi: 10.1101/ gad.233676.113.

Daniels M, Dhokia V, Richard-Parpaillon L, Ohnuma S-I. 2004. Identification of Xenopus cyclin-dependent kinase inhibitors, p16Xic2 and p17Xic3. Gene 342:41-47. doi: 10.1016/j.gene.2004.07.038.

Deflorian G, Tiso N, Ferretti E, Meyer D, Blasi F, Bortolussi M, Argenton F. 2004. Prep1.1 has essential genetic functions in hindbrain development and cranial neural crest cell differentiation. Development 131:613-627. doi: 10.1242/dev.00948.

Dominguez-Sola D, Ying CY, Grandori C, Ruggiero L, Chen B, Li M, Galloway DA, Gu W, Gautier J, DallaFavera R. 2007. Non-transcriptional control of DNA replication by c-Myc. Nature 448:445-451. doi: 10. 1038/nature05953.

Donley N, Thayer MJ. 2013. DNA replication timing, genome stability and cancer: late and/or delayed DNA replication timing is associated with increased genomic instability. Seminars in Cancer Biology 23:80-89. doi: 10. 1016/j.semcancer.2013.01.001.

El Yakoubi W, Borday C, Hamdache J, Parain K, Tran HT, Vleminckx K, Perron M, Locker M. 2012. Hes4 controls proliferative properties of neural stem cells during retinal ontogenesis. Stem Cells 30:2784-2795. doi: 10.1002/ stem.1231.

Fernandez-Diaz LC, Laurent A, Girasoli S, Turco M, Longobardi E, lotti G, Jenkins NA, Fiorenza MT, Copeland NG, Blasi F. 2010. The absence of Prep1 causes p53-dependent apoptosis of mouse pluripotent epiblast cells. Development 137:3393-3403. doi: 10.1242/dev.050567.

Ferretti E, Villaescusa JC, Di Rosa P, Fernandez-Diaz LC, Longobardi E, Mazzieri R, Miccio A, Micali N, Selleri L, Ferrari G, Blasi F. 2006. Hypomorphic mutation of the TALE gene Prep1 (pKnox1) causes a major reduction of $\mathrm{Pbx}$ and Meis proteins and a pleiotropic embryonic phenotype. Molecular and Cellular Biology 26:5650-5662. doi: 10.1128/MCB.00313-06.

Gee ST, Milgram SL, Kramer KL, Conlon FL, Moody S. 2011. Yes-associated protein 65 (YAP) expands neural progenitors and regulates Pax3 expression in the neural plate border zone. PLOS ONE 6:e20309. doi: 10.1371/ journal.pone.0020309.

Imajo M, Ebisuya M, Nishida E. 2015. Dual role of YAP and TAZ in renewal of the intestinal epithelium. Nature Cell Biology 17:7-19. doi: 10.1038/ncb3084.

Insinga A, Cicalese A, Pelicci PG. 2014. DNA damage response in adult stem cells. Blood Cells, Molecules \& Diseases 52:147-151. doi: 10.1016/j.bcmd.2013.12.005.

lotti G, Longobardi E, Masella S, Dardaei L, De Santis F, Micali N, Blasi F. 2011. Homeodomain transcription factor and tumor suppressor Prep1 is required to maintain genomic stability. Proceedings of the National Academy of Sciences of USA 108:E314. doi: 10.1073/pnas.1105216108.

Jansson L, Larsson J. 2012. Normal hematopoietic stem cell function in mice with enforced expression of the Hippo signaling effector YAP1. PLOS ONE 7:e32013. doi: 10.1371/journal.pone.0032013.

Kanekar S, Perron M, Dorsky R, Harris WA, Jan LY, Jan YN, Vetter ML. 1997. Xath5 participates in a network of bHLH genes in the developing Xenopus retina. Neuron 19:981-994. doi: 10.1016/S0896-6273(00)80391-8.

Koberna K, Ligasová A, Malínský J, Pliss A, Siegel AJ, Cvacková Z, Fidlerová H, Masata M, Fialová M, Raska I, Berezney R. 2005. Electron microscopy of DNA replication in 3-D: evidence for similar-sized replication foci throughout S-phase. Journal of Cellular Biochemistry 94:126-138. doi: 10.1002/jcb.20300. 
Lian I, Kim J, Okazawa H, Zhao J, Zhao B, Yu J, Chinnaiyan A, Israel MA, Goldstein LS, Abujarour R, Ding S, Guan KL. 2010. The role of YAP transcription coactivator in regulating stem cell self-renewal and differentiation. Genes \& Development 24:1106-1118. doi: 10.1101/gad.1903310.

Locker M, Agathocleous M, Amato MA, Parain K, Harris WA, Perron M. 2006. Hedgehog signaling and the retina: insights into the mechanisms controlling the proliferative properties of neural precursors. Genes \& Development 20:3036. doi: 10.1101/gad.391106.

Micali N, Ferrai C, Fernandez-Diaz LC, Blasi F, Crippa MP. 2009. Prep1 directly regulates the intrinsic apoptotic pathway by controlling Bcl-XL levels. Molecular and Cellular Biology 29:1143-1151. doi: 10.1128/MCB.01273-08.

Miesfeld JB, Link BA. 2014. Establishment of transgenic lines to monitor and manipulate Yap/Taz-Tead activity in zebrafish reveals both evolutionarily conserved and divergent functions of the Hippo pathway. Mechanisms of Development 133:177-188. doi: 10.1016/j.mod.2014.02.003.

Naye F, Tréguer K, Soulet F, Faucheux C, Fédou S, Thézé N, Thiébaud P. 2007. Differential expression of two TEF-1 (TEAD) genes during Xenopus laevis development and in response to inducing factors. The International Journal of Developmental Biology 51:745-752. doi: 10.1387/ijdb.072375fn.

Neto-Silva RM, de Beco S, Johnston LA. 2010. Evidence for a growth-stabilizing regulatory feedback mechanism between Myc and Yorkie, the Drosophila homolog of Yap. Developmental Cell 19:507-520. doi: 10.1016/j. devcel.2010.09.009.

Nowakowski RS, Lewin SB, Miller MW. 1989. Bromodeoxyuridine immunohistochemical determination of the lengths of the cell cycle and the DNA-synthetic phase for an anatomically defined population. Journal of Neurocytology 18:311-318. doi: 10.1007/BF01190834.

Ohashi K, Mizuno K. 2014. A novel pair of split venus fragments to detect protein-protein interactions by in vitro and in vivo bimolecular fluorescence complementation assays. Methods in Molecular Biology 1174:247-262.

Pefani D-E, Latusek R, Pires I, Grawenda AM, Yee KS, Hamilton G, van der Weyden L, Esashi F, Hammond EM, O'Neill E. 2014. RASSF1A-LATS1 signalling stabilizes replication forks by restricting CDK2-mediated phosphorylation of BRCA2. Nature Cell Biology 16:962-971. doi: 10.1038/ncb3035.

Peng HW, Slattery M, Mann RS. 2009. Transcription factor choice in the Hippo signaling pathway: homothorax and yorkie regulation of the microRNA bantam in the progenitor domain of the Drosophila eye imaginal disc. Genes \& Development 23:2307-2319. doi: 10.1101/gad.1820009.

Perron M. 2003. A novel function for Hedgehog signalling in retinal pigment epithelium differentiation. Development 130:1565-1577. doi: 10.1242/dev.00391.

Perron M, Kanekar S, Vetter ML, Harris WA. 1998. The genetic sequence of retinal development in the ciliary margin of the Xenopus eye. Developmental Biology 199:185-200. doi: 10.1006/dbio.1998.8939.

Piccolo S, Dupont S, Cordenonsi M. 2014. The biology of YAP/TAZ: hippo signaling and beyond. Physiological Reviews 94:1287-1312. doi: 10.1152/physrev.00005.2014.

Quastler H, Sherman FG. 1959. Cell population kinetics in the intestinal epithelium of the mouse. Experimental Cell Research 17:420-438. doi: 10.1016/0014-4827(59)90063-1.

Ramos A, Camargo FD. 2012. The Hippo signaling pathway and stem cell biology. Trends in Cell Biology 1-8. doi: 10.1016/j.tcb.2012.04.006.

Robinson K, Asawachaicharn N, Galloway DA, Grandori C. 2009. c-Myc accelerates S-phase and requires WRN to avoid replication stress. PLOS ONE 4:e5951. doi: 10.1371/journal.pone.0005951.

Rogakou EP, Pilch DR, Orr AH, Ivanova VS, Bonner WM. 1998. DNA double-stranded breaks induce histone H2AX phosphorylation on serine 139. The Journal of Biological Chemistry 273:5858-5868. doi: 10.1074/jbc.273. 10.5858.

Saka Y, Hagemann Al, Smith JC. 2008. Visualizing protein interactions by bimolecular fluorescence complementation in Xenopus. Methods 45:192-195. doi: 10.1016/j.ymeth.2008.06.005.

Shen Z, Stanger BZ. 2015. YAP Regulates S-Phase Entry in Endothelial Cells. PLOS ONE 10:e0117522. doi: 10. 1371/journal.pone.0117522.

Srinivasan SV, Dominguez-Sola D, Wang LC, Hyrien O, Gautier J. 2013. Cdc45 is a critical effector of mycdependent DNA replication stress. Cell Reports 3:1629-1639. doi: 10.1016/j.celrep.2013.04.002.

Stocker H. 2011. Growth control: Myc and Yorkie get connected. Current Biology 21:R37-R39. doi: 10.1016/j.cub. 2010.11.057.

Tallafuss a, Gibson D, Morcos P, Li Y, Seredick S, Eisen J, Washbourne P. 2012. Turning gene function ON and OFF using sense and antisense photo-morpholinos in zebrafish. Development 139:1691-1699. doi: 10.1242/dev.072702.

Turrero García M, Chang Y, Arai Y, Huttner WB. 2015. S-phase duration is the main target of cell cycle regulation in neural progenitors of developing ferret neocortex. The Journal of Comparative Neurology. doi: 10.1002/cne.23801.

van Dierendonck JH, Keyzer R, van de Velde CJ, Cornelisse CJ. 1989. Subdivision of S-phase by analysis of nuclear 5-bromodeoxyuridine staining patterns. Cytometry 10:143-150. doi: 10.1002/cyto.990100205.

Vogelstein B, Lane D, Levine AJ. 2000. Surfing the p53 network. Nature 408:307-310. doi: 10.1038/35042675.

Watanabe Y, Maekawa M. 2010. Spatiotemporal regulation of DNA replication in the human genome and its association with genomic instability and disease. Current Medicinal Chemistry 17:222-233. doi: 10.2174/ 092986710790149756.

Wetts R, Serbedzija GN, Fraser SE. 1989. Cell lineage analysis reveals multipotent precursors in the ciliary margin of the frog retina. Developmental Biology 136:254-263. doi: 10.1016/0012-1606(89)90146-2.

Xiao W, Wang J, Ou C, Zhang Y, Ma L, Weng W, Pan Q, Sun F. 2013. Mutual interaction between YAP and c-Myc is critical for carcinogenesis in liver cancer. Biochemical and Biophysical Research Communications 439:167-172. doi: 10.1016/j.bbrc.2013.08.071. 
Xue XY, Harris WA. 2011. Using myc genes to search for stem cells in the ciliary margin of the Xenopus retina. Developmental Neurobiology 72:475-490. doi: 10.1002/dneu.20887.

Yamazaki S, Hayano M, Masai H. 2013. Replication timing regulation of eukaryotic replicons: Rif1 as a global regulator of replication timing. Trends in Genetics 29:449-460. doi: 10.1016/j.tig.2013.05.001.

Zeman MK, Cimprich KA. 2014. Causes and consequences of replication stress. Nature Cell Biology 16:2-9. doi: 10.1038/ncb2897.

Zhang H, Deo M, Thompson RC, Uhler MD, Turner DL. 2012. Negative regulation of Yap during neuronal differentiation. Developmental Biology 361:103-115. doi: 10.1016/j.ydbio.2011.10.017.

Zhang T, Zhou Q, Pignoni F. 2011. Yki/YAP, Sd/TEAD and Hth/MEIS control tissue specification in the Drosophila eye disc epithelium. PLOS ONE 6:e22278. doi: 10.1371/journal.pone.0022278.

Zhang W, Nandakumar N, Shi Y, Manzano M, Smith A, Graham G, Gupta S, Vietsch EE, Laughlin SZ, Wadhwa M, Chetram M, Joshi M, Wang F, Kallakury B, Toretsky J, Wellstein A, Yi C. 2014. Downstream of mutant KRAS, the transcription regulator YAP is essential for neoplastic progression to pancreatic ductal adenocarcinoma. Science Signaling 7:ra42. doi: 10.1126/scisignal.2005049.

Zhao B, Wei X, Li W, Udan RS, Yang Q, Kim J, Xie J, Ikenoue T, Yu J, Li L, Zheng P, Ye K, Chinnaiyan A, Halder G, Lai ZC, Guan KL. 2007. Inactivation of YAP oncoprotein by the Hippo pathway is involved in cell contact inhibition and tissue growth control. Genes \& Development 21:2747-2761. doi: 10.1101/gad.1602907.

Zhao R, Fallon TR, Saladi SV, Pardo-Saganta A, Villoria J, Mou H, Vinarsky V, Gonzalez-Celeiro M, Nunna N, Hariri LP, Camargo F, Ellisen LW, Rajagopal J. 2014. Yap tunes airway epithelial size and architecture by regulating the identity, maintenance, and self-renewal of stem cells. Developmental Cell 30:151-165. doi: 10.1016/j.devcel. 2014.06.004. 